\title{
Solving the Boltzmann equation deterministically by the Fast Spectral Method: application to gas microflows
}

\author{
Lei $\mathbf{W u}^{1}$, Jason M Reese ${ }^{2}$, and Yonghao Zhang ${ }^{1}$ \\ ${ }^{1}$ James Weir Fluids Laboratory, Department of Mechanical and Aerospace Engineering, \\ University of Strathclyde, Glasgow G1 1XJ, UK \\ ${ }^{2}$ School of Engineering, University of Edinburgh, Edinburgh EH9 3JL, UK
}

(Received ?; revised ?; accepted ?. - To be entered by editorial office)

Based on the fast spectral approximation to the Boltzmann collision operator, we present an accurate and efficient deterministic numerical method for solving the Boltzmann equation. First, the linearised Boltzmann equation is solved for Poiseuille and thermal creep flows, where the influence of different molecular models on the mass and heat flow rates is assessed, and the Onsager-Casimir relation at the microscopic level for large Knudsen numbers is demonstrated. Recent experimental measurements of mass flow rates along a rectangular tube with large aspect ratio are compared with numerical results for the linearised Boltzmann equation. Then, a number of two-dimensional microflows in the transition and free molecular flow regimes are simulated using the nonlinear Boltzmann equation. The influence of the molecular model is discussed, as well as the applicability of the linearised Boltzmann equation. For thermally driven flows in the free molecular regime, it is found that the magnitudes of the flow velocity are inversely proportional to the Knudsen number. The streamline patterns of thermal creep flow inside a closed rectangular channel are analysed in detail: when the Knudsen number is smaller than a critical value, the flow pattern can be predicted based on a linear superposition of the velocity profiles of linearised Poiseuille and thermal creep flows between parallel plates. For large Knudsen numbers, the flow pattern can be determined using the linearised Poiseuille and thermal creep velocity profiles at the critical Knudsen number. The critical Knudsen number is found to be related to the aspect ratio of the rectangular channel.

Key words: Authors should not enter keywords on the manuscript

\section{Introduction}

The Knudsen number $K n$, the ratio of the molecular mean free path $\lambda$ to the characteristic flow length $\ell$, is an important parameter in rarefied gas dynamics. The NavierStokes-Fourier (NSF) equations based on the continuum fluid hypothesis can usually be used up to $K n \sim 0.001 \dagger$. When $K n$ is larger, the continuum hypothesis breaks down and the NSF equations fail to capture the non-conventional behaviour of the rarefied flow. This situation is most frequently encountered in high-altitude aerodynamics and in the vacuum industry (where $\lambda$ is large), and in micro/nano-electromechanical systems (where

$\dagger$ There are some exceptions where the NSF equations do not describe the gas dynamics properly even when $K n \rightarrow 0$. For example, the ghost effect induced by a spatially periodic variation of the wall temperature, see Y. Sone, Kinetic Theory and Fluid Dynamics (Birkhauser Boston, 2002). 
$\ell$ is small). The Boltzmann equation (BE) is a fundamental model at the microscopic level describing rarefied gas dynamics for the full range of Knudsen number (Cercignani 1990). It uses the velocity distribution function (VDF) defined in a six-dimensional phase space to describe the system state, along with the Boltzmann collision operator (BCO) to model the intermolecular interactions. The BE is complicated, which makes it highly desirable to use macroscopic equations like the NSF model. In the past, Burnett models and Grad moment equations have been derived from the BE. While the Burnett models are intrinsically unstable (Garcia-Colin et al. 2008), regularised Grad moment equations have been applied up to the transition flow regime for some specific problems $(\mathrm{Gu} \&$ Emerson 2009; Rana et al. 2013).

For moderately or highly rarefied gases, it is necessary to solve the BE numerically. The direct simulation Monte Carlo (DSMC) method (Bird 1994) is the prevailing technique. It is efficient for high speed flows, but becomes computationally time-consuming in microflow simulations where the flow velocity is far smaller than the thermal velocity. To tackle this difficulty, information-preservation (Fan \& Shen 2001; Masters \& Ye 2007) and low-noise (Baker \& Hadjiconstantinou 2005; Homolle \& Hadjiconstantinou 2007; Radtke et al. 2011) DSMC methods have been proposed. The information-preserving method introduces information quantities (such as the information velocity and information temperature) to reduce the statistical noise, which has proven highly effective. However, its convergence to the BE has not been rigorously shown. The low-noise DSMC method significantly improves the computational efficiency of the original DSMC method by simulating only the deviation from the equilibrium state. To our knowledge, for microflow simulations, it is currently the most efficient stochastic method to solve the BE (Radtke et al. 2011).

The deterministic numerical solution of the BE is, however, theoretically the best method to resolve small signals, because it is not subject to fluctuations. However, the high computational cost in the approximation of the BCO restricts the number of discrete velocity grids one can use; this does not cause problems when the VDF is smooth, but it does lead to failure at large $K n$ where discontinuities and/or fine structures exist. Therefore, a deterministic numerical method allowing a large number of discrete velocity grids but with reduced computational cost is needed for highly rarefied gas flow simulations.

The Fast Spectral Method (FSM), proposed by Mouhot \& Pareschi (2006), and improved by $\mathrm{Wu}$ et al. (2013), is one such method. The FSM handles the binary molecular collisions in frequency space instead of velocity space, where the Fourier spectrum of the BCO appears as a convolved sum of the weighted spectrum of the VDF. When this sum is carried out directly, the computational cost is $O\left(N_{\xi}^{6}\right)$, where $N_{\xi}$ is the number of frequency components in one direction. The main idea of the FSM is to separate the frequency components in the weighted function, so that the convolution theorem can be applied, reducing the computational cost to $O\left(M^{2} N_{\xi}^{3} \log N_{\xi}\right)$, where the number of discrete angles $M$ is far less than $N_{\xi}$. This separation requires special forms of the collision kernel. In our previous paper (Wu et al. 2013), these special forms were constructed and validated, making the FSM applicable to all inverse power-law (IPL) potentials (except for the Coulomb potential) and the Lennard-Jones (LJ) potential.

In this present paper we explore the features of the FSM for microflow simulation. We assess the influence of the discrete velocity grids, the number of frequency components, and the number of discrete angles $M$ on the accuracy of the FSM. Based on this accurate and efficient numerical method, a number of two-dimensional microflows are simulated, and the influence of the molecular models is discussed. The asymptotic behaviour of thermally driven flows in the free molecular regime is investigated and, specifically, the flow pattern of the thermal creep flow inside a closed rectangular channel is studied in 
detail. A new criterion and method is proposed to predict the flow pattern in closed rectangular channels of arbitrary aspect ratio and Knudsen number.

The paper is organized as follows: the BE with special anisotropic collision kernels is introduced in Sec. 2, and the approximation of the (nonlinear and linear) BCOs by the FSM is presented in Sec. 3. In Sec. 4, based on the linearised BE, the numerical accuracy of the FSM is evaluated and its computational efficiency is demonstrated for both Poiseuille and thermal creep flows. The influence of molecular models on the flow rates is discussed and the accuracy of the special collision kernel for the LJ potential is evaluated. In Sec. 5, based on the nonlinear BE, a number of two-dimensional flows are simulated and the influence of the molecular models is compared. A new scaling law for the flow velocity is proposed for thermally driven flows in the free molecular regime. The flow field of the thermal creep flow inside a closed rectangular channel is also investigated. Finally, we conclude this paper in Sec. 6 .

\section{The Boltzmann equation}

\subsection{The nonlinear Boltzmann equation}

In this paper we consider a monatomic gas. In reality, the intermolecular interaction is best described by the (6-12) LJ potential. For simplicity, however, IPL molecular models have been introduced, and are widely used by researchers. Hence we first consider IPL intermolecular potentials. According to the Chapman-Enskog expansion (Chapman \& Cowling 1970), the shear viscosity $\mu$ is proportional to $T^{\omega}$, with $T$ being the temperature and $\omega$ the viscosity index. Special collision kernels can be used to recover not only the value of the shear viscosity but also its temperature dependence (Wu et al. 2013). We therefore consider the BE

where the $\mathrm{BCO}$ is given by

$$
\frac{\partial f}{\partial t}+\mathbf{v} \cdot \frac{\partial f}{\partial \mathbf{x}}=Q\left(f, f_{*}\right)
$$

$$
Q\left(f, f_{*}\right) \equiv \iint B(|\mathbf{u}|, \theta)\left(f_{*}^{\prime} f^{\prime}-f_{*} f\right) d \Omega d \mathbf{v}_{*},
$$

with the following form of the collision kernel

$$
B(|\mathbf{u}|, \theta)=\frac{|\mathbf{u}|^{\alpha}}{K} \sin ^{\alpha+\gamma-1}\left(\frac{\theta}{2}\right) \cos ^{-\gamma}\left(\frac{\theta}{2}\right),
$$

where $\alpha=2(1-\omega)$ and $\gamma$ is a free parameter.

The $\mathrm{BE}$ above is given in dimensionless form: the spatial variable $\mathbf{x}$ is normalised by a characteristic flow length $\ell$; the molecular velocity $\mathbf{v}$ and relative velocity $\mathbf{u}$ are normalised by the most probable molecular speed $v_{m}=\sqrt{2 k_{B} T_{0} / m}$, with $k_{B}$ the Boltzmann constant, $T_{0}$ the reference temperature, and $m$ the molecular mass. The time $t$ is normalised by $\ell / v_{m}$, while the VDF is normalised by $n_{0} / v_{m}^{3}$, where $n_{0}$ is the reference molecular number density. The subscript $*$ represents the second molecule in the binary collision, the superscript ' stands for quantities after the collision, and $\Omega$ is the solid angle. Finally, to recover the shear viscosity, we have

$$
K=\frac{2^{7-\omega}}{5} \Gamma\left(\frac{\alpha+\gamma+3}{2}\right) \Gamma\left(2-\frac{\gamma}{2}\right) K n,
$$

where

$$
K n=\frac{\mu\left(T_{0}\right)}{n_{0} \ell} \sqrt{\frac{\pi}{2 m k_{B} T_{0}}},
$$


is the unconfined Knudsen number and $\Gamma$ is the gamma function. Note that the rarefaction parameter $K n$ is $15 \pi / 2(7-2 \omega)(5-2 \omega)$ times larger than the Knudsen number $K n_{v h s}$, where $\lambda$ is defined by equation (4.52) in the book by Bird (1994). Also note that sometimes the parameter $\delta$ is used (Sharipov \& Seleznev 1994, 1998), which is related to the unconfined Knudsen number $K n$ by

$$
\delta=\frac{\sqrt{\pi}}{2 K n} .
$$

For realistic (6-12) LJ potentials, the shear viscosity from the Chapman-Enskog expansion is not a power-law function of temperature; only in a small temperature range could the viscosity be described by a single power-law function of temperature. For instance, for helium and argon, in the temperature range $293<T<373$, it has been suggested to use $\omega=0.66$ and $\omega=0.81$, respectively (Chapman \& Cowling 1970; Bird 1994). For a broader temperature range, the single IPL model may not work well. Note that if we use the realistic collision kernel given by Sharipov \& Bertoldo (2009a), the calculation of the weighted function becomes very complicated and, moreover, the efficiency of the FSM is reduced by one order of magnitude. Therefore, we propose using the following collision kernel

$$
B(|\mathbf{u}|, \theta)=\frac{5 \sum_{j=1}^{3} b_{j}\left(k_{B} T_{0} / 2 \epsilon\right)^{\left(\alpha_{j}-1\right) / 2} \sin ^{\alpha_{j}-1}(\theta / 2)|\mathbf{u}|^{\alpha_{j}} / \Gamma\left(\frac{\alpha_{j}+3}{2}\right)}{64 \sqrt{2} K n \sum_{j=1}^{3} b_{j}\left(k_{B} T_{0} / \epsilon\right)^{\left(\alpha_{j}-1\right) / 2}},
$$

to approximate that of the realistic (6-12) LJ potential (Wu et al. 2013), where $b_{1}=407.4$, $b_{2}=-811.9, b_{3}=414.4, \alpha_{1}=0.2, \alpha_{2}=0.1, \alpha_{3}=0$, and $\epsilon$ is the potential depth. This expression can recover the shear viscosity for the LJ potential over the temperature range $1<k_{B} T / \epsilon<25$, and produce accurate macroscopic quantities and microscopic VDFs in normal shock waves when compared to both experimental data and molecular dynamics simulations (Wu et al. 2013). Hereafter, the BE using the approximated collision kernel (2.7) will be called the LJ model, and the accuracy of this model in the microflow simulations will be assessed.

\subsection{The linearised Boltzmann equation}

If the state of the gas is weakly nonequilibrium, the nonlinear BE (2.1) can be linearised. We express the VDF then as

$$
f(t, \mathbf{x}, \mathbf{v})=f_{e q}+h(t, \mathbf{x}, \mathbf{v})
$$

where

$$
f_{e q}(\mathbf{v})=\frac{\exp \left(-|\mathbf{v}|^{2}\right)}{\pi^{3 / 2}}
$$

is the global equilibrium velocity distribution function, and $h(t, \mathbf{x}, \mathbf{v})$ represents the deviation from global equilibrium satisfying $|h| \ll 1$. The nonlinear BE is then linearised to

where

$$
\frac{\partial h}{\partial t}+\mathbf{v} \cdot \frac{\partial h}{\partial \mathbf{x}}=\mathcal{L}_{g}(h)-\nu_{e q}(v) h,
$$

$$
\mathcal{L}_{g}(h)=\iint B(|\mathbf{u}|, \theta)\left[f_{e q}\left(\mathbf{v}^{\prime}\right) h\left(\mathbf{v}_{*}^{\prime}\right)+f_{e q}\left(\mathbf{v}_{*}^{\prime}\right) h\left(\mathbf{v}^{\prime}\right)-f_{e q}(\mathbf{v}) h\left(\mathbf{v}_{*}\right)\right] d \Omega d \mathbf{v}_{*},
$$

which can be viewed as a linear gain term, and

$$
\nu_{e q}(v)=\iint B(|\mathbf{u}|, \theta) f_{e q}\left(\mathbf{v}_{*}\right) d \Omega d \mathbf{v}_{*}
$$


is the equilibrium collision frequency.

\section{The Fast Spectral Method}

The five-fold integral BCO poses a challenge for the numerical solution of the BE. Unlike the discrete velocity method, which handles the binary collision in velocity space, the FSM works in a corresponding frequency space, where the VDF and BCO are expanded in Fourier series. The discrete velocity grid points can be nonuniform to capture discontinuities, however, in order to take advantage of fast Fourier transform (FFT)-based convolution, the frequency components should be uniformly distributed, i.e.

$$
\begin{aligned}
f(\mathbf{v}) & =\sum_{\mathbf{j}=-\mathbf{N} / 2}^{\mathbf{N} / 2-1} \hat{f}_{\mathbf{j}} \exp \left(i \xi_{\mathbf{j}} \cdot \mathbf{v}\right), \\
\hat{f}_{\mathbf{j}} & =\frac{1}{V_{\mathcal{D}}} \int_{\mathcal{D}} f(\mathbf{v}) \exp \left(-i \xi_{\mathbf{j}} \cdot \mathbf{v}\right) d \mathbf{v},
\end{aligned}
$$

where $i$ is the imaginary unit, $\mathbf{N}=\left(N_{1}^{\prime}, N_{2}^{\prime}, N_{3}^{\prime}\right)$, the equidistant frequency components are $\xi_{\mathbf{j}}=\mathbf{j} \pi / L$ with $\mathbf{j}=\left(j_{1}, j_{2}, j_{3}\right)$ and $L$ being the maximum truncated velocity, and $\mathcal{D}$ is the truncated velocity domain with $V_{\mathcal{D}}$ its volume. The velocity domain is discretized by $N_{1} \times N_{2} \times N_{3}$ points and the spectrum $\hat{f}$ of the VDF can be calculated numerically by the trapezoidal rule. Note that the number of velocity grid points is usually larger than the number of frequency components.

The BCO and its Fourier spectrum $\hat{Q}$ are also expanded by Fourier series. The $\mathbf{j}$-th Fourier mode of the BCO is related to the spectrum $\hat{f}$ as follows (Wu et al. 2013):

$$
\hat{Q}(\mathbf{j})=\frac{1}{V_{\mathcal{D}}} \int_{\mathcal{D}} Q\left(f, f_{*}\right) \exp \left(-i \xi_{\mathbf{j}} \cdot \mathbf{v}\right) d \mathbf{v}=\sum_{\substack{1+\mathbf{m}=\mathbf{j} \\ 1, \mathbf{m}=-\mathbf{N} / 2}}^{\mathbf{N} / 2-1} \hat{f}_{\mathbf{l}} \hat{f}_{\mathbf{m}}[\beta(\mathbf{l}, \mathbf{m})-\beta(\mathbf{m}, \mathbf{m})],
$$

where $\mathbf{l}=\left(l_{1}, l_{2}, l_{3}\right), \mathbf{m}=\left(m_{1}, m_{2}, m_{3}\right)$, and $\beta(\mathbf{l}, \mathbf{m})$ is the weighted function. For the collision kernel (2.3) for IPL potentials, the frequency components $\xi_{1}$ and $\xi_{\mathbf{m}}$ in the weighted function can be separated as

$$
\beta(\mathbf{l}, \mathbf{m})_{I P L} \simeq \frac{4}{K} \sum_{p, q=1}^{M} \psi_{\gamma}\left\{\sqrt{\left|\xi_{\mathbf{m}}\right|^{2}-\left(\xi_{\mathbf{m}} \cdot \mathbf{e}_{\theta_{p}, \varphi_{q}}\right)^{2}}\right\} \omega_{p} \omega_{q} \sin \theta_{p} \phi_{\alpha+\gamma}\left(\xi_{\mathbf{l}} \cdot \mathbf{e}_{\theta_{p}, \varphi_{q}}\right),
$$

while for the collision kernel (2.7) to approximate the LJ potential, we have

$$
\begin{aligned}
\beta(\mathbf{l}, \mathbf{m})_{L J} \simeq & \frac{5}{16 \sqrt{2} K n \sum_{j=1}^{3} b_{j}\left(k_{B} T_{0} / \epsilon\right)^{\left(\alpha_{j}-1\right) / 2}} \sum_{p, q=1}^{M} \psi_{\gamma}\left\{\sqrt{\left|\xi_{\mathbf{m}}\right|^{2}-\left(\xi_{\mathbf{m}} \cdot \mathbf{e}_{\theta_{p}, \varphi_{q}}\right)^{2}}\right\} \\
& \times \omega_{p} \omega_{q} \sin \theta_{p} \sum_{j=1}^{3} b_{j}\left(k_{B} T_{0} / 2 \epsilon\right)^{\left(\alpha_{j}-1\right) / 2} \phi_{\alpha_{j}}\left(\xi_{\mathbf{l}} \cdot \mathbf{e}_{\theta_{p}, \varphi_{q}}\right) / \Gamma\left(\frac{\alpha_{j}+3}{2}\right),
\end{aligned}
$$

where $\theta_{p}\left(\varphi_{q}\right)$ and $\omega_{p}\left(\omega_{q}\right)$ are the $p(q)$-th point and weight in the Gauss-Legendre quadrature, respectively, with $\theta, \varphi \in[0, \pi] ; \mathbf{e}_{\theta_{p}, \varphi_{q}}=\left(\sin \theta_{p} \cos \varphi_{q}, \sin \theta_{p} \sin \varphi_{q}, \cos \theta_{p}\right)$, $\phi_{\alpha+\gamma}(s)=2 \int_{0}^{R} \rho^{\alpha+\gamma} \cos (\rho s) d \rho$, and $\psi_{\gamma}(s)=2 \pi \int_{0}^{R} \rho^{1-\gamma} J_{0}(\rho s) d \rho$. Here $R=2 \sqrt{2} L /(2+$ $\sqrt{2})$ is chosen approximately as the average value of its minimum allowed value $2 \sqrt{2} L /(3+$ $\sqrt{2}$ ) and its maximum allowed value $L$ (see figure $5 \mathrm{in} \mathrm{Wu}$ et al. (2013)), and $J_{0}$ is the zeroth-order Bessel function. 
For conventional spectral methods (Pareschi \& Russo 2000; Gamba \& Tharkabhushanam 2009), equation (3.2) is calculated by direct sum, with a computational cost $O\left(N_{1}^{\prime 2} N_{2}^{\prime 2} N_{3}^{\prime 2}\right)$. However, if the FFT-based convolution is applied, the computational cost is reduced to $O\left(M^{2} N_{1}^{\prime} N_{2}^{\prime} N_{3}^{\prime} \log \left(N_{1}^{\prime} N_{2}^{\prime} N_{3}^{\prime}\right)\right)$. The number of discrete angles $M$ controls the computational cost and the numerical accuracy. It will be shown below that $M=6$ produces sufficiently accurate results. Hence the FSM is significantly faster than conventional spectral methods. Note that the computational cost of the IPL and LJ models are exactly the same.

When the spectrum of the $\mathrm{BCO}$ is obtained, we calculate the $\mathrm{BCO}$ through the following equation:

$$
Q\left(f, f_{*}\right)=\sum_{\mathbf{j}=-\mathbf{N} / 2}^{\mathbf{N} / 2-1} \hat{Q}(\mathbf{j}) \exp \left(i \xi_{\mathbf{j}} \cdot \mathbf{v}\right) .
$$

Now we consider the fast spectral approximation of the linearised collision operator. The equilibrium collision frequency can be calculated analytically. For the linearised gain term $\mathcal{L}_{g}$, when the IPL potential is considered, the $\mathbf{j}$-th Fourier mode of $\mathcal{L}_{g}$ is

$$
\begin{aligned}
\hat{\mathcal{L}}_{g}(\mathbf{j}) \approx & \frac{4}{K} \sum_{p, q=1}^{M} \sum_{\substack{1+\mathbf{m}=\mathbf{j} \\
\mathbf{1} \mathbf{m}=-\mathbf{N} / 2}}^{\mathbf{N} / 2-1} \omega_{p} \omega_{q}\left[\hat{f}_{e q}(\mathbf{l}) \phi_{\alpha+\gamma}\left(\xi_{\mathbf{l}}, \theta_{p}, \varphi_{q}\right)\right] \cdot\left[\hat{h}_{\mathbf{m}} \psi_{\gamma}\left(\xi_{\mathbf{m}}, \theta_{p}, \varphi_{q}\right)\right] \cdot \sin \theta_{p} \\
& +\frac{4}{K} \sum_{p, q=1}^{M} \sum_{\substack{1+\mathbf{m}=\mathbf{j} \\
\mathbf{l} \mathbf{m}=\mathbf{N} / 2}}^{\mathbf{N} / 2-1} \omega_{p} \omega_{q}\left[\hat{h}_{\mathbf{l}} \phi_{\alpha+\gamma}\left(\xi_{\mathbf{l}}, \theta_{p}, \varphi_{q}\right)\right] \cdot\left[\hat{f}_{e q}(\mathbf{m}) \psi_{\gamma}\left(\xi_{\mathbf{m}}, \theta_{p}, \varphi_{q}\right)\right] \cdot \sin \theta_{p} \\
& -\frac{4}{K} \sum_{\substack{1+\mathbf{m}=\mathbf{j} \\
\mathbf{1}, \mathbf{m}=-\mathbf{N} / 2}}^{\mathbf{N} / 2-1} \hat{f}_{e q}(\mathbf{l}) \cdot\left[\hat{h}_{\mathbf{m}} \phi_{\text {loss }}\right]
\end{aligned}
$$

where $\phi_{\text {loss }}=\sum_{p, q=1}^{M} \omega_{p} \omega_{q} \phi_{\alpha+\gamma}\left(\xi_{\mathbf{m}}, \theta_{p}, \varphi_{q}\right) \psi_{\gamma}\left(\xi_{\mathbf{m}}, \theta_{p}, \varphi_{q}\right) \sin \theta_{p}$ and $\hat{h}$ is the spectrum of the VDF $h$. For the LJ collision kernel (2.7), the $\mathbf{j}$-th Fourier mode of the linear gain term can be obtained in a similar way.

Note that each term on the right hand side of equation (3.6) is a convolution; like equation (3.2), these can be calculated effectively by a FFT-based convolution. Since the Fourier transform of the terms $\hat{f}_{e q}(\mathbf{l}) \phi_{\alpha+\gamma}\left(\xi_{\mathbf{l}}, \theta_{p}, \varphi_{q}\right)$ and $\hat{f}_{e q}(\mathbf{m}) \psi_{\gamma}\left(\xi_{\mathbf{m}}, \theta_{p}, \varphi_{q}\right)$ can be precomputed and stored, the computational time required for the linearised collision operator is nearly the same as that for the full BCO. However, for IPL potentials, if $\gamma=(1-\alpha) / 2$, the linear gain operator is simplified to

$$
\mathcal{L}_{g}(h)=\iint B(|u|, \theta)\left[2 f_{e q}\left(\mathbf{v}^{\prime}\right) h\left(\mathbf{v}_{*}^{\prime}\right)-f_{e q}(\mathbf{v}) h\left(\mathbf{v}_{*}\right)\right] d \Omega d \mathbf{v}_{*},
$$

and its $\mathbf{j}$-th Fourier mode is approximated by

$$
\begin{aligned}
\hat{\mathcal{L}}_{g}(\mathbf{j}) \approx & \frac{8}{K} \sum_{p, q=1}^{M} \sum_{\substack{1+\mathbf{m}=\mathbf{j} \\
1, \mathbf{m}=-\mathbf{N} / 2}}^{\mathbf{N} / 2-1} \omega_{p} \omega_{q} \hat{f}_{e q}(\mathbf{l}) \phi_{\alpha+\gamma}\left(\xi_{\mathbf{l}}, \theta_{p}, \varphi_{q}\right) \hat{h}_{\mathbf{m}} \psi_{\gamma}\left(\xi_{\mathbf{m}}, \theta_{p}, \varphi_{q}\right) \sin \theta_{p} \\
& -\frac{4}{K} \sum_{\substack{1+\mathbf{m}=\mathbf{j} \\
\mathbf{1}, \mathbf{m}=\mathbf{N} / 2}}^{\mathbf{N} / 2-1} \hat{f}_{e q}(\mathbf{l}) \cdot\left[\hat{h}_{\mathbf{m}} \phi_{\text {loss }}\right]
\end{aligned}
$$

so the computational cost can be reduced by half. 
The symmetry in the VDF can also help to reduce computational cost further. For example, if $h$ is symmetric with respect to $v_{3}$, i.e., $h\left(v_{3}\right)=h\left(-v_{3}\right)$, then $q$ in equation (3.8) can only take values of $1,2, \cdots, M / 2$ for even $M$. We denote the spectrum obtained in this way as $\hat{\mathcal{L}}_{g}^{z}(\mathbf{j})$ and let $\mathcal{L}_{g}^{z}(\mathbf{v})=\sum_{\mathbf{j}} \hat{\mathcal{L}}_{g}^{z}(\mathbf{j}) \exp \left(i \xi_{\mathbf{j}} \cdot \mathbf{v}\right)$. Then the linear gain operator $\mathcal{L}_{g}(\mathbf{v})$ can be obtained as $\mathcal{L}_{g}^{z}\left(v_{1}, v_{2}, v_{3}\right)+\mathcal{L}_{g}^{z}\left(v_{1}, v_{2},-v_{3}\right)$. In this way the computational cost is reduced by half. This technique can also be applied to the nonlinear BCO.

\section{Numerical results for Poiseuille and thermal creep flows using the linearised Boltzmann equation}

Poiseuille and thermal creep flows are two classical problems in rarefied gas dynamics. Because of the singular (over-concentration) behaviour in the VDF (Takata \& Funagane 2011), the numerical simulation of a highly rarefied gas is a difficult task; for a long time accurate numerical results have been limited to $K n \leqslant 20$ for the hard sphere gas (Ohwada et al. 1989; Doi 2010). Recently, some progress has been achieved both analytically and numerically in obtaining the mass and heat flow rates at large Knudsen numbers (Takata \& Funagane 2011; Funagane \& Takata 2012; Doi 2012a,b). Here, based on the FSM for the linearised $\mathrm{BCO}$, we solve these two classical flows in parallel plate and rectangular tube configurations up to $K n \sim 10^{6}$. The accuracy of the FSM for solving the linearised BE is evaluated by comparing our numerical results for the Poiseuille flow of a hard sphere gas with those obtained by the numerical kernel method (Ohwada et al. 1989). We can then determine the discretization resolutions required in the velocity and frequency spaces, as well as the number of discrete angles $M$. The influence of the molecular models on the mass and heat flow rates is discussed below; specifically, we check the model accuracy by comparing flow rates for the IPL and LJ models with those for the realistic LJ potentials presented in Sharipov \& Bertoldo (2009b). Finally, the recent experimental data by Ewart et al. (2007) is evaluated.

\subsection{Poiseuille flow between parallel plates}

Consider a gas between two parallel plates located at $x_{2}=-\ell / 2$ and $\ell / 2$, respectively. A uniform pressure gradient is imposed on the gas in the $x_{1}$ direction: the pressure is given by $n_{0} k_{B} T_{0}\left(1+\beta_{P} x_{1} / \ell\right)$ with $\left|\beta_{P}\right| \ll 1$. The $\mathrm{BE}$ is linearised around the equilibrium state at rest with number density $n_{0}$ and temperature $T_{0}$, where the VDF is expressed as $f=f_{e q}+\beta_{P}\left(x_{1} f_{e q}+h\right)$. The linearised $\mathrm{BE}$ in the dimensionless form is then

$$
v_{2} \frac{\partial h}{\partial x_{2}}=\mathcal{L}_{g}(h)-\nu_{e q}(v) h-v_{1} f_{e q},
$$

and the velocity and heat flux are $V_{1}=\int v_{1} h d \mathbf{v}$ and $q_{1}=\int\left(|\mathbf{v}|^{2}-\frac{5}{2}\right) v_{1} h d \mathbf{v}$, respectively.

Due to symmetry, only half of the spatial region $\left(-0.5 \leqslant x_{2} \leqslant 0\right)$ is simulated, with a specular-reflection boundary condition at $x_{2}=0$. The diffuse boundary condition is adopted at the wall, i.e., $h\left(x_{2}=-0.5, v_{2}>0\right)=0$. The spatial domain is divided into $N_{s}$ nonuniform sections, with most of the discrete points placed near the wall:

$$
x_{2}=\left(10-15 s+6 s^{2}\right) s^{3}-0.5,
$$

where $s=\left(0,1, \cdots, N_{s}\right) / 2 N_{s}$. For $N_{s}=100$, the size of the smallest section is $1.24 \times 10^{-6}$, while the largest is 0.0094 .

Because of the symmetry and smoothness of the VDF, $N_{1}, N_{3}=12$ uniform grids are used in the $v_{1}(>0)$ and $v_{3}(>0)$ directions. The maximum molecular velocity is at $L=6$. 


\begin{tabular}{|c|c|c|c|c|c|c|c|c|}
\hline \multirow[b]{3}{*}{$k$} & \multicolumn{6}{|c|}{ Fast spectral method } & \multicolumn{2}{|c|}{ Literature } \\
\hline & \multicolumn{2}{|c|}{$M=6$} & \multicolumn{2}{|c|}{$M=8$} & \multicolumn{2}{|c|}{$M=12$} & & \\
\hline & & $\mathcal{Q}$ & & $\mathcal{Q}$ & $-\mathcal{M}$ & $\mathcal{Q}$ & $-\mathcal{M}$ & $\mathcal{Q}$ \\
\hline 0.1 & 1.1 & & 953 & & & & 1.1930 & 0.0553 \\
\hline 0.15 & & 0.0758 & & & & & & \\
\hline 0.2 & 0.9006 & 0.0 & 0.9003 & & & & & \\
\hline 0.3 & 0.8156 & & 0.8154 & & & & & \\
\hline 0.4 & 0.7804 & & 0.7802 & & & & & \\
\hline 0.6 & 0.7566 & 0.1727 & 0.7563 & 0.1729 & & & 0.7562 & \\
\hline 0.8 & 0.7537 & 0.1957 & 0.7534 & 0.15 & & 0.1 & 0.7533 & \\
\hline 1 & 0.758 & 0.2139 & 0.7576 & & & & 0.7574 & 0.2140 \\
\hline 1.5 & & & & & & & & 0.2477 \\
\hline 2 & & & & & & & & \\
\hline 3 & & & & & & & & \\
\hline 4 & & & & & 0.8 & & 0.8 & \\
\hline 6 & & & 0.9325 & & 0.9 & & 0.9321 & \\
\hline 8 & & 0.40 & 81 & 0.40 & 0.9 & & 0.97 & 0.4015 \\
\hline 10 & 1.0161 & & & & & & & 0.4242 \\
\hline 15 & 1.090 & & 1.0908 & & 1.09 & & 1.0908 & 0.4669 \\
\hline 20 & 1.1475 & 0.4977 & 1.1477 & 0.4982 & 1.1475 & 0.4978 & 1.1479 & 0.4984 \\
\hline $10^{2}$ & 1.5139 & 0.6897 & 1.5141 & 0.6898 & 1.5142 & 0.6900 & 1.5143 & 0.6900 \\
\hline $10^{3}$ & 2.1208 & 0.9959 & 2.1209 & 0.9959 & 2.1210 & 0.9960 & 2.1210 & 0.9960 \\
\hline $10^{4}$ & 2.7614 & 1.3165 & 2.7614 & & 2.7615 & & 2.7615 & 1.3166 \\
\hline $10^{5}$ & 3.4094 & 1.6406 & 3.4094 & 1.6405 & 3.4094 & 1.6405 & 3.4094 & 1.6406 \\
\hline $10^{6}$ & 4.0586 & 1.9653 & 4.0587 & 1.9652 & 4.0587 & 1.9652 & 4.0587 & 1.9652 \\
\hline
\end{tabular}

Table 1: Mass and heat flow rates in Poiseuille flow between parallel plates of a hard sphere gas. For $k=8 K n / 5 \sqrt{\pi} \leqslant 20$ and $k \geqslant 10^{2}$, the data in the last two columns are collected from Ohwada et al. (1989) and Takata \& Funagane (2011), respectively.

In the discretization of $v_{2}, N_{2}$ nonuniform grids are used:

$$
v_{2}=\frac{L_{2}}{\left(N_{2}-1\right)^{2}}\left(-N_{2}+1,-N_{2}+3, \cdots, N_{2}-1\right)^{2},
$$

where $L_{2}=4$ and $\imath$ is a positive odd number. Due to the over-concentration in the VDF, large values of $N_{s}$ and $\imath$ should be chosen when investigating large $K n$ problems.

The number of frequency components in the $\xi_{1}$ and $\xi_{3}$ directions are $N_{1}^{\prime} N_{3}^{\prime}=24 \times 24$, and there are $N_{2}^{\prime}$ frequency components in the $\xi_{2}$ direction. The FFT is used in the $v_{1}$ and $v_{3}$ directions, while in the $v_{2}$ direction the direct sum is implemented $\dagger$, resulting in an overall computational cost of $O\left(N_{2} N_{2}^{\prime} N_{1}^{\prime} N_{3}^{\prime} \ln \left(N_{1}^{\prime} N_{3}^{\prime}\right)\right)$, which is comparable to the FFT-based convolution sum of equation (3.8).

To obtain the stationary solution, the following implicit iteration scheme is used:

$$
\nu_{e q}(v) h^{k+1}+v_{2} \frac{\partial h^{k+1}}{\partial x_{2}}=\mathcal{L}_{g}\left(h^{k}\right)-v_{1} f_{e q},
$$

where $\partial h / \partial x_{2}$ is approximated by a second-order upwind finite difference. The calculation of $\mathcal{L}_{g}\left(h^{k}\right)$ is as follows: when $h^{k}$ is known, we obtain $\hat{\mathcal{L}}_{g}$ from equation (3.8). Then we

$\dagger$ For nonuniform velocity grids (4.3), we use $\sum_{m} g\left(v_{2 m}\right) w_{m}$ to approximate $\int g\left(v_{2}\right) d v_{2}$, where $w_{m}=\imath L_{2} m^{\imath-1} /\left(N_{2}-1\right)^{\imath}$ with $m \in\left[-N_{2}+1,-N_{2}+3, \cdots, N_{2}-1\right]$. 


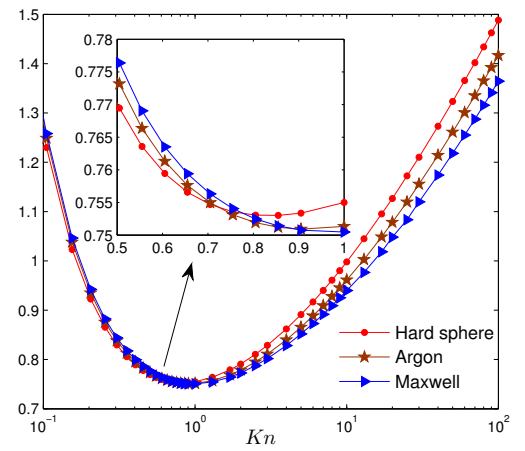

(a) Mass flow rate $-\mathcal{M}$ at $\alpha_{a}=1.0$

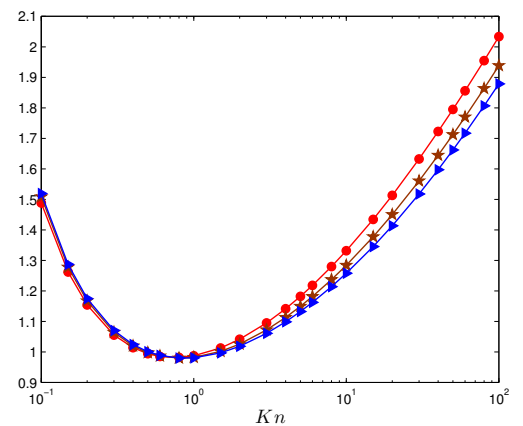

(c) Mass flow rate $-\mathcal{M}$ at $\alpha_{a}=0.8$

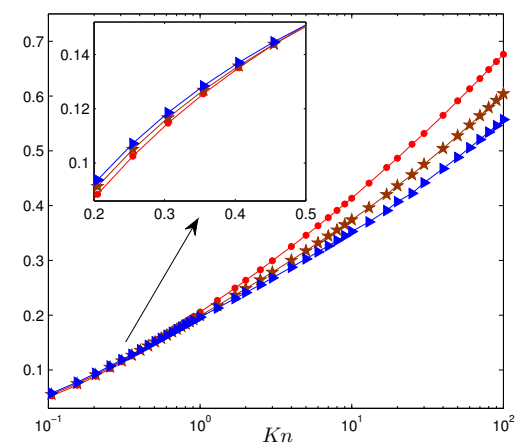

(b) Heat flow rate $\mathcal{Q}$ at $\alpha_{a}=1.0$

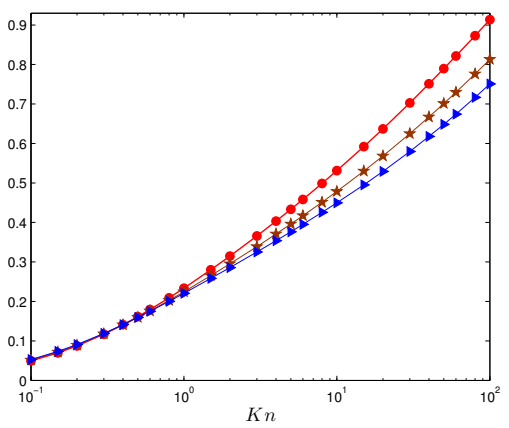

(d) Heat flow rate $\mathcal{Q}$ at $\alpha_{a}=0.8$

Figure 1: Comparisons of mass and heat flow rates for different IPL molecular models in Poiseuille gas flow between parallel plates. Here $\alpha_{a}$ represents the wall accommodation coefficient, where the boundary condition at $x_{2}=-0.5$ is $h\left(v_{1}, v_{2}, v_{3}, x_{2}=-0.5\right)=$ $\left(1-\alpha_{a}\right) h\left(v_{1},-v_{2}, v_{3}, x_{2}=-0.5\right)$ for $v_{2}>0$.

obtain $\mathcal{L}_{g}\left(h^{k}\right)$ by applying the inverse FFT to $\hat{\mathcal{L}}_{g}: \mathcal{L}_{g}\left(h^{k}\right)=\sum_{\mathbf{j}=-\mathbf{N} / 2}^{\mathbf{N} / 2-1} \hat{\mathcal{L}}_{g}(j) \exp \left(i \xi_{\mathbf{j}} \cdot \mathbf{v}\right)$. The iterations are terminated when changes in the mass flow rate

$$
\mathcal{M}=2 \int_{-1 / 2}^{0} V_{1} d x_{2}
$$

and heat flow rate

$$
\mathcal{Q}=2 \int_{-1 / 2}^{0} q_{1} d x_{2}
$$

between two consecutive iteration steps are less than $10^{-8}$.

To validate the FSM for the linearised BE, our numerical results are compared to those obtained using the numerical kernel method (Ohwada et al. 1989) for a hard sphere gas $(\omega=0.5)$. For $k=8 K n / 5 \sqrt{\pi} \leqslant 20$, the number of equispaced frequency components is $N_{2}^{\prime}=32$ and the velocity discretization is given by equation (4.3) with $N_{2}=48$ and $\imath=3$. For $k \geqslant 100$, we choose $N_{2}^{\prime}=64, N_{2}=100$, and $\imath=7$. The comparisons are summarised in Table 1. It is seen that, when we use $M=6$, the relative error in the mass flow rate between the two methods is less than $0.1 \%$, while that in the heat flow rate is less than $0.25 \%$. When $M=8$ (or 12), a relative error less than $0.01 \%$ can be achieved. Our method produces accurate results even when the Knudsen number is very large. We also compared our numerical results with those from the information-preserving DSMC (Fan 


\begin{tabular}{|c|c|c|c|c|c|c|c|c|c|c|c|c|c|}
\hline \multirow[b]{2}{*}{$\delta$} & \multicolumn{3}{|c|}{$\mathrm{He}$} & \multicolumn{2}{|c|}{$\mathrm{Ne}$} & \multicolumn{3}{|c|}{$\mathrm{Ar}$} & \multicolumn{2}{|c|}{$\mathrm{Kr}$} & \multicolumn{3}{|c|}{$\mathrm{Xe}$} \\
\hline & $\begin{array}{l}\text { IPL } \\
\text { FSM }\end{array}$ & $\begin{array}{c}\text { LJ } \\
\text { FSM }\end{array}$ & $\begin{array}{l}\text { LJ } \\
\text { SB }\end{array}$ & $\begin{array}{c}\text { LJ } \\
\text { FSM }\end{array}$ & $\begin{array}{l}\text { LJ } \\
\text { SB }\end{array}$ & $\begin{array}{l}\text { IPL } \\
\text { FSM }\end{array}$ & $\begin{array}{c}\text { LJ } \\
\text { FSM }\end{array}$ & $\begin{array}{l}\text { LJ } \\
\text { SB }\end{array}$ & $\begin{array}{c}\text { LJ } \\
\text { FSM }\end{array}$ & $\begin{array}{l}\text { LJ } \\
\text { SB }\end{array}$ & $\begin{array}{l}\text { IPL } \\
\text { FSM }\end{array}$ & $\begin{array}{c}\text { LJ } \\
\text { FSM }\end{array}$ & $\begin{array}{l}\text { LJ } \\
\text { SB }\end{array}$ \\
\hline 0.010 & 2.852 & 2.814 & 2.668 & 2.733 & 2.581 & 2.788 & 2.615 & 2.502 & 2.577 & 2.495 & 2.770 & 2.562 & 2.497 \\
\hline 0.020 & 2.534 & 2.507 & 2.424 & 2.444 & 2.358 & 2.478 & 2.349 & 2.280 & 2.318 & 2.269 & 2.463 & 2.306 & 2.270 \\
\hline 0.025 & 2.438 & 2.414 & 2.345 & 2.357 & 2.287 & 2.385 & 2.270 & 2.211 & 2.242 & 2.199 & 2.371 & 2.231 & 2.199 \\
\hline 0.040 & 2.246 & 2.230 & 2.182 & 2.185 & 2.140 & 2.200 & 2.115 & 2.072 & 2.092 & 2.058 & 2.188 & 2.082 & 2.057 \\
\hline 0.050 & 2.160 & 2.148 & 2.107 & 2.109 & 2.073 & 2.117 & 2.046 & 2.009 & 2.025 & 1.995 & 2.106 & 2.017 & 1.993 \\
\hline 0.100 & 1.920 & 1.917 & 1.893 & 1.894 & 1.876 & 1.889 & 1.853 & 1.830 & 1.838 & 1.816 & 1.881 & 1.832 & 1.813 \\
\hline 0.200 & 1.726 & 1.728 & 1.715 & 1.717 & 1.707 & 1.704 & 1.691 & 1.679 & 1.681 & 1.668 & 1.699 & 1.677 & 1.665 \\
\hline 0.250 & 1.674 & 1.677 & 1.667 & 1.668 & 1.661 & 1.656 & 1.647 & 1.637 & 1.638 & 1.628 & 1.651 & 1.635 & 1.62 \\
\hline 0.400 & 1.584 & 1.587 & 1.582 & 1.583 & 1.580 & 1.572 & 1.569 & 1.566 & 1.564 & 1.559 & 1.569 & 1.561 & 1.55 \\
\hline 0.500 & 1.552 & 1.555 & 1.552 & 1.551 & 1.550 & 1.542 & 1.541 & 1.540 & 1.537 & 1.535 & 1.540 & 1.536 & 1.533 \\
\hline 1.000 & 1.504 & 1.505 & 1.507 & 1.505 & 1.508 & 1.502 & 1.504 & 1.507 & 1.504 & 1.507 & 1.502 & 1.504 & 1.507 \\
\hline 1.600 & 1.530 & 1.530 & 1.534 & 1.531 & 1.536 & 1.533 & 1.536 & 1.540 & 1.538 & 1.543 & 1.534 & 1.539 & 1.544 \\
\hline 2.000 & 1.565 & 1.565 & 1.570 & 1.566 & 1.572 & 1.570 & 1.573 & 1.578 & 1.576 & 1.582 & 1.572 & 1.578 & 1.583 \\
\hline 2.500 & 1.619 & 1.618 & 1.624 & 1.620 & 1.626 & 1.626 & 1.629 & 1.634 & 1.633 & 1.639 & 1.628 & 1.635 & 1.641 \\
\hline 4.000 & 1.813 & 1.811 & 1.819 & 1.814 & 1.822 & 1.824 & 1.827 & 1.833 & 1.833 & 1.839 & 1.827 & 1.836 & 1.842 \\
\hline 5.000 & 1.955 & 1.953 & 1.963 & 1.957 & 1.966 & 1.968 & 1.971 & 1.978 & 1.978 & 1.985 & 1.972 & 1.981 & 1.988 \\
\hline 10.00 & 2.723 & 2.721 & 2.740 & 2.726 & 2.743 & 2.744 & 2.746 & 2.756 & 2.755 & 2.764 & 2.748 & 2.759 & 2.768 \\
\hline
\end{tabular}

Table 2: Mass flow rate $(-2 \mathcal{M})$ in Poiseuille flow of various gases between parallel plates for various $\delta$, see equation (2.6). The data in columns denoted by SB are those results from Sharipov \& Bertoldo (2009b).

\& Shen 2001), and found that the information-preserving method works well except at extremely large Knudsen number: the relative error in the mass flow rate between the two methods reaches about $7 \%$ at $K n_{v h s}=100$.

We now compare the mass and heat flow rates for a hard sphere gas $(\omega=0.5)$, for argon ( $\omega=0.81)$, and for a Maxwell gas $(\omega=1)$ using the IPL models. The numerical results are shown in figure 1 . We denote $K n_{c}(\approx 0.9)$ the Knudsen number at which the Knudsen minimum in the mass flow rate exists. When $K n>K n_{c}$ (or $K n<K n_{c}$ ), the mass flow rate decreases (or increases) as the viscosity index $\omega$ increases, for a fixed value of $K n$. For instance, at $K n=10$, the mass flow rate of the Maxwell gas is about $94 \%$ that of the hard sphere gas when $\alpha_{a}=1$. The underlying mechanism for this may be understood in terms of the effective collision frequency: for the same value of shear viscosity, the average collision frequency $\int \nu_{e q}(v) f_{e q} d v / \int f_{e q} d v$ increases with $\omega$ (see figures 12 and 13 in $\mathrm{Wu}$ et al. (2013) and the corresponding text therein). Therefore, Maxwell molecules have a greater effective collision frequency (and a smaller effective $K n$ ) than hard sphere molecules. Since at large $K n$ the mass flow rate increases with $K n$, the Maxwell gas has a lower mass flow rate than the hard sphere gas. Conversely, since at small $K n$ the mass flow rate decreases with $K n$, when $K n<K n_{c}$, the Maxwell gas has a higher mass flow rate than the hard sphere gas, although the difference is very small. The heat flow rate behaves similarly to the mass flow rate; that is, when $K n>0.5$ (or $K n<0.5$ ), the heat flow rate decreases (or increases) as $\omega$ increases, for a fixed value of $K n$. The difference in flow rates between various gases with the same value of shear viscosity holds when the wall accommodation coefficient $\alpha_{a}$ is not 1 , see figure $1(\mathrm{c}, \mathrm{d})$.

Since IPL potentials are simplifications of the realistic LJ potential, it is interesting to check the accuracy of the collision kernels (2.3) and (2.7). Tables 2 and 3 compare 


\begin{tabular}{|c|c|c|c|c|c|c|c|c|c|c|c|c|c|}
\hline \multirow[b]{2}{*}{$\delta$} & \multicolumn{3}{|c|}{$\mathrm{He}$} & \multicolumn{2}{|c|}{$\mathrm{Ne}$} & \multicolumn{3}{|c|}{$\mathrm{Ar}$} & \multicolumn{2}{|c|}{$\mathrm{Kr}$} & \multicolumn{3}{|c|}{$\mathrm{Xe}$} \\
\hline & $\begin{array}{l}\text { IPL } \\
\text { FSM }\end{array}$ & $\begin{array}{c}\text { LJ } \\
\text { FSM }\end{array}$ & $\begin{array}{l}\text { LJ } \\
\text { SB }\end{array}$ & $\begin{array}{c}\text { LJ } \\
\text { FSM }\end{array}$ & $\begin{array}{l}\text { LJ } \\
\text { SB }\end{array}$ & $\begin{array}{l}\text { IPL } \\
\text { FSM }\end{array}$ & $\begin{array}{c}\text { LJ } \\
\text { FSM }\end{array}$ & $\begin{array}{l}\text { LJ } \\
\text { SB }\end{array}$ & $\begin{array}{c}\text { LJ } \\
\text { FSM }\end{array}$ & $\begin{array}{l}\text { LJ } \\
\text { SB }\end{array}$ & $\begin{array}{l}\text { IPL } \\
\text { FSM }\end{array}$ & $\begin{array}{c}\text { LJ } \\
\text { FSM }\end{array}$ & $\begin{array}{l}\text { LJ } \\
\text { SB }\end{array}$ \\
\hline 0.010 & 1.252 & 1.217 & & & & & & & & & & & 1.056 \\
\hline 0.020 & 1.092 & 1.066 & 1.027 & 1.006 & 0.990 & 1.033 & 0.924 & 0.937 & 0.899 & 0.933 & 1.016 & 0.890 & 0.935 \\
\hline 0.025 & 1.042 & 1.020 & 0.989 & 0.965 & 0.954 & 0.986 & 0.890 & 0.900 & 0.866 & 0.895 & 0.970 & 0.857 & 0.897 \\
\hline 0.040 & 0.942 & 0.927 & 0.909 & 0.884 & 0.878 & 0.893 & 0.821 & 0.824 & 0.801 & 0.818 & 0.879 & 0.793 & 0.819 \\
\hline 0.050 & 0.897 & 0.885 & 0.870 & 0.847 & 0.843 & 0.851 & 0.790 & 0.790 & 0.771 & 0.782 & 0.838 & 0.764 & 0.783 \\
\hline 0.100 & 0.764 & 0.761 & 0.751 & 0.737 & 0.734 & 0.729 & 0.697 & 0.689 & 0.682 & 0.680 & 0.719 & 0.677 & 0.679 \\
\hline 0.200 & 0.641 & 0.644 & 0.637 & 0.631 & 0.627 & 0.617 & 0.604 & 0.595 & 0.593 & 0.585 & 0.610 & 0.589 & 0.583 \\
\hline 0.250 & 0.604 & 0.607 & 0.601 & 0.597 & 0.593 & 0.583 & 0.574 & 0.565 & 0.564 & 0.556 & 0.577 & 0.560 & 0.554 \\
\hline 0.400 & 0.527 & 0.531 & 0.526 & 0.525 & 0.521 & 0.513 & 0.509 & 0.503 & 0.502 & 0.495 & 0.509 & 0.499 & 0.493 \\
\hline 0.500 & 0.492 & 0.495 & 0.491 & 0.491 & 0.487 & 0.480 & 0.477 & 0.473 & 0.473 & 0.467 & 0.477 & 0.470 & 0.465 \\
\hline 1.000 & 0.386 & 0.387 & 0.385 & 0.386 & 0.383 & 0.381 & 0.381 & 0.379 & 0.379 & 0.377 & 0.380 & 0.379 & 0.376 \\
\hline 1.600 & 0.316 & 0.316 & 0.315 & 0.316 & 0.314 & 0.315 & 0.316 & 0.315 & 0.316 & 0.315 & 0.315 & 0.316 & 0.315 \\
\hline 2.000 & 0.283 & 0.284 & 0.282 & 0.284 & 0.282 & 0.284 & 0.285 & 0.284 & 0.286 & 0.285 & 0.284 & 0.286 & 0.286 \\
\hline 2.500 & 0.252 & 0.252 & 0.251 & 0.252 & 0.251 & 0.254 & 0.255 & 0.254 & 0.256 & 0.256 & 0.254 & 0.257 & 0.256 \\
\hline 4.000 & 0.189 & 0.189 & 0.188 & 0.190 & 0.189 & 0.192 & 0.193 & 0.193 & 0.195 & 0.196 & 0.193 & 0.196 & 0.197 \\
\hline 5.000 & 0.162 & 0.162 & 0.161 & 0.163 & 0.162 & 0.166 & 0.166 & 0.166 & 0.168 & 0.169 & 0.167 & 0.169 & 0.170 \\
\hline 10.00 & 0.094 & 0.093 & 0.093 & 0.094 & 0.093 & 0.097 & 0.097 & 0.097 & 0.099 & 0.099 & 0.098 & 0.100 & 0.100 \\
\hline
\end{tabular}

Table 3: Heat flow rate $(2 \mathcal{Q})$ in Poiseuille flow of various gases between parallel plates for various $\delta$. The data in columns denoted by SB are those results from Sharipov \& Bertoldo (2009b).
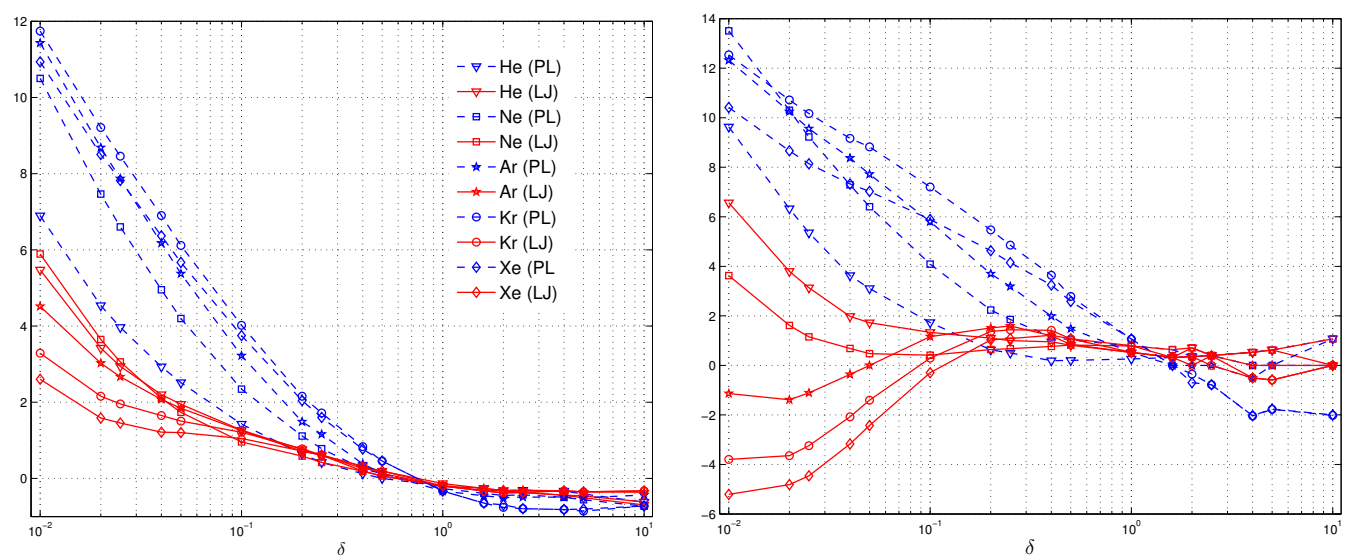

Figure 2: Relative differences in the mass (left) and heat (right) flow rates between the molecular models and LJ potentials in Poiseuille gas flow between parallel plates for various $\delta$.

the mass flow rates and heat flow rates for five noble gases with the data of Sharipov \& Bertoldo $(2009 b)$ for the LJ potential. For the IPL models, at a temperature of 300K, we use the viscosity index $\omega_{H e}=\omega_{N e}=0.66$ (so only the results for helium are shown), $\omega_{A r}=0.81 \simeq \omega_{K r}=0.80$ (so only the results for argon are shown), and $\omega_{X e}=0.85$. For the LJ potential, the potential depth $\epsilon$ of $\mathrm{He}, \mathrm{Ne}, \mathrm{Ar}, \mathrm{Kr}$, and Xe are $10.22 k_{B}, 35.7 k_{B}$, $124 k_{B}, 190 k_{B}$, and $229 k_{B}$, respectively. The relative differences between the mass and 


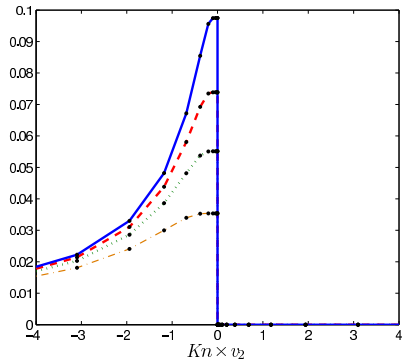

(a) At $x_{2}=-0.5$

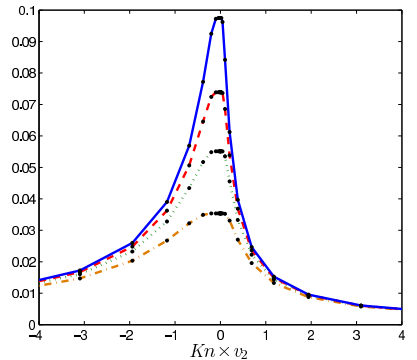

(b) At $x_{2}=-0.25$

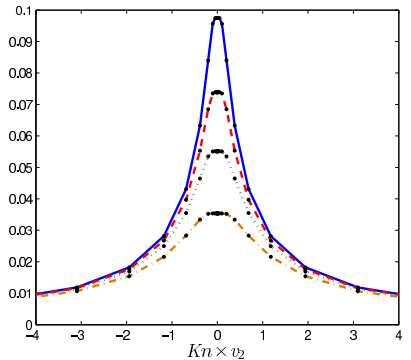

(c) At $x_{2}=0$

Figure 3: Demonstration of the Onsager-Casimir relation at the microscopic level. Lines (or dots): velocity distribution functions $\left(|v|^{2}-\frac{5}{2}\right) h_{P}$ in Poiseuille flow (or $h_{T}$ in thermal creep flow) at $v_{1}, v_{3}=6 / 31$ and $K n=10^{4}$. From top to bottom, the curves correspond to the hard sphere, helium (IPL), argon (IPL), and Maxwell gases, respectively.

heat flow rates produced by the FSM and those in Sharipov \& Bertoldo (2009b) are visualized in figure 2. For $\delta>0.2$, the relative difference $\left|\mathcal{M}_{I P L}-\mathcal{M}_{S B}\right| /\left|\mathcal{M}_{S B}\right|$ between the IPL models and LJ potentials is less than $1 \%$. For $\delta<0.2$, the relative difference increases as $\delta$ decreases. Specifically, at $\delta=0.01$, the IPL model overestimates the mass flow rate by around $6.9 \%, 10.5 \%, 11.4 \%, 11.7 \%$, and $11.0 \%$ for $\mathrm{He}, \mathrm{Ne}, \mathrm{Ar}, \mathrm{Kr}$, and Xe, respectively. When the collision kernel (2.7) is used for the LJ potential, at $\delta=0.01$, the LJ model overestimates the mass flow rate by around $5.5 \%, 6.0 \%, 4.5 \%, 3.3 \%$, and $2.6 \%$ for $\mathrm{He}, \mathrm{Ne}, \mathrm{Ar}, \mathrm{Kr}$, and Xe, respectively. As $\delta$ increases, the relative difference quickly decreases. Similar behaviour can be observed for the heat flow rate, except that the LJ models for Ar, Kr, and Xe underestimate the heat flow rate at small values of $\delta$. Generally speaking, when compared to the solution for the realistic LJ potential, the LJ model yields closer results than the IPL model.

\subsection{Thermal creep flow between parallel plates}

In thermal creep flow, the wall temperature varies as $T=T_{0}\left(1+\beta_{T} x_{1} / \ell\right)$, where $\left|\beta_{T}\right| \ll 1$. The VDF is expressed as $f=f_{e q}+\beta_{T}\left[x_{1} f_{e q}\left(|\mathbf{v}|^{2}-\frac{5}{2}\right)+h\right]$ and the linearised BE is given by equation (4.1) with the last term replaced by $v_{1}\left(|\mathbf{v}|^{2}-\frac{5}{2}\right) f_{\text {eq }}$.

The Onsager-Casimir relation (Loyalka 1971; Sharipov 1994a,b; Takata $2009 a, b$ ) states that the mass flow rate in thermal creep flow is equal to the heat flow rate in Poiseuille flow: $\int V_{1}\left[h_{T}\right] d x_{2}=\int q_{1}\left[h_{P}\right] d x_{2}$. Recently, Takata \& Funagane (2011) observed a strong relation, that is, at large $K n, V_{1}\left[h_{T}\right]$ and $q_{1}\left[h_{P}\right]$ have identical spatial profiles, i.e., $V_{1}\left[h_{T}\right]=q_{1}\left[h_{P}\right]+O\left[K n^{-1}(\ln K n)^{2}\right]$. The VDFs obtained by our FSM in figure 3 show that a stronger Onsager-Casimir relation exists at the microscopic level, that is, $h_{T} \approx$ $\left(|\mathbf{v}|^{2}-\frac{5}{2}\right) h_{P}$, at large $K n$, for various IPL models.

Because of the Onsager-Casimir relation, we compare only the heat flow rates in thermal creep flow for different potential models. The results are in Table 4. For a particular molecular model, the heat flow rate increase monotonically as $\delta$ decreases. As in the Poiseuille flow case, different molecular models have different flow rates at the same value of shear viscosity; for IPL models, the heat flow rate always decreases as $\omega$ increases.

The relative difference in heat flow rates is visualized in figure 4 . At $\delta=0.01$, for He, $\mathrm{Ne}, \mathrm{Ar}, \mathrm{Kr}$, and Xe, the IPL models overestimate the heat flow rate relative to Sharipov \& Bertoldo $(2009 b)$ by $6.6 \%, 10.3 \%, 11.4 \%, 11.7 \%$, and $11 \%$, while the LJ model overestimate the heat flow rate by $5.1 \%, 5.7 \%, 4.6 \%, 3.5 \%$, and and $2.9 \%$, respectively. As $\delta$ increases, the relative difference quickly decreases. This comparison, together with those 


\begin{tabular}{|c|c|c|c|c|c|c|c|c|c|c|c|c|c|}
\hline \multirow[b]{2}{*}{$\delta$} & \multicolumn{3}{|c|}{$\mathrm{He}$} & \multicolumn{2}{|c|}{$\mathrm{Ne}$} & \multicolumn{3}{|c|}{$\mathrm{Ar}$} & \multicolumn{2}{|c|}{$\mathrm{Kr}$} & \multicolumn{3}{|c|}{$\mathrm{Xe}$} \\
\hline & $\begin{array}{l}\text { IPL } \\
\text { FSM }\end{array}$ & $\begin{array}{c}\text { LJ } \\
\text { FSM }\end{array}$ & $\begin{array}{l}\text { LJ } \\
\text { SB }\end{array}$ & $\begin{array}{c}\text { LJ } \\
\text { FSM }\end{array}$ & $\begin{array}{l}\text { LJ } \\
\text { SB }\end{array}$ & $\begin{array}{l}\text { IPL } \\
\text { FSM }\end{array}$ & $\begin{array}{c}\text { LJ } \\
\text { FSM }\end{array}$ & $\begin{array}{l}\text { LJ } \\
\text { SB }\end{array}$ & $\begin{array}{c}\text { LJ } \\
\text { FSM }\end{array}$ & $\begin{array}{l}\text { LJ } \\
\text { SB }\end{array}$ & $\begin{array}{l}\text { IPL } \\
\text { FSM }\end{array}$ & $\begin{array}{c}\text { LJ } \\
\text { FSM }\end{array}$ & $\begin{array}{l}\text { LJ } \\
\text { SB }\end{array}$ \\
\hline 0.010 & 6.269 & 6.182 & 5.879 & 6.010 & 5.684 & 6.140 & 5.768 & 5.512 & 5.691 & 5.496 & 6.104 & 5.662 & 5.500 \\
\hline 0.020 & 5.496 & 5.435 & 5.263 & 5.301 & 5.121 & 5.385 & 5.109 & 4.958 & 5.048 & 4.934 & 5.354 & 5.024 & 4.935 \\
\hline 0.025 & 5.255 & 5.202 & 5.059 & 5.082 & 4.936 & 5.151 & 4.906 & 4.779 & 4.850 & 4.754 & 5.121 & 4.828 & 4.754 \\
\hline 0.040 & 4.761 & 4.725 & 4.626 & 4.632 & 4.542 & 4.670 & 4.491 & 4.404 & 4.445 & 4.376 & 4.644 & 4.427 & 4.373 \\
\hline 0.050 & 4.532 & 4.505 & 4.421 & 4.424 & 4.353 & 4.448 & 4.298 & 4.225 & 4.257 & 4.197 & 4.424 & 4.240 & 4.193 \\
\hline 0.100 & 3.848 & 3.840 & 3.792 & 3.793 & 3.761 & 3.784 & 3.709 & 3.669 & 3.679 & 3.641 & 3.766 & 3.666 & 3.635 \\
\hline 0.200 & 3.196 & 3.200 & 3.174 & 3.175 & 3.162 & 3.151 & 3.120 & 3.103 & 3.098 & 3.080 & 3.138 & 3.089 & 3.074 \\
\hline 0.250 & 2.992 & 2.997 & 2.977 & 2.977 & 2.968 & 2.951 & 2.929 & 2.918 & 2.909 & 2.897 & 2.940 & 2.901 & 2.891 \\
\hline 0.400 & 2.569 & 2.575 & 2.562 & 2.562 & 2.560 & 2.538 & 2.526 & 2.525 & 2.511 & 2.508 & 2.529 & 2.504 & 2.502 \\
\hline 0.500 & 2.371 & 2.377 & 2.367 & 2.367 & 2.366 & 2.343 & 2.335 & 2.337 & 2.321 & 2.322 & 2.336 & 2.316 & 2.317 \\
\hline 1.000 & 1.772 & 1.776 & 1.770 & 1.770 & 1.771 & 1.752 & 1.750 & 1.756 & 1.741 & 1.745 & 1.748 & 1.737 & 1.741 \\
\hline 1.600 & 1.387 & 1.391 & 1.385 & 1.387 & 1.387 & 1.373 & 1.371 & 1.377 & 1.365 & 1.369 & 1.369 & 1.362 & 1.366 \\
\hline 2.000 & 1.216 & 1.219 & 1.213 & 1.215 & 1.216 & 1.203 & 1.202 & 1.207 & 1.197 & 1.201 & 1.200 & 1.194 & 1.198 \\
\hline 2.500 & 1.054 & 1.057 & 1.051 & 1.054 & 1.053 & 1.043 & 1.042 & 1.046 & 1.038 & 1.041 & 1.040 & 1.036 & 1.039 \\
\hline 4.000 & 0.752 & 0.754 & 0.750 & 0.752 & 0.752 & 0.745 & 0.745 & 0.747 & 0.742 & 0.744 & 0.743 & 0.740 & 0.743 \\
\hline 5.000 & 0.631 & 0.633 & 0.629 & 0.631 & 0.630 & 0.625 & 0.625 & 0.627 & 0.622 & 0.625 & 0.623 & 0.621 & 0.624 \\
\hline 10.00 & 0.347 & 0.348 & 0.345 & 0.347 & 0.346 & 0.344 & 0.344 & 0.345 & 0.343 & 0.344 & 0.343 & 0.342 & 0.344 \\
\hline
\end{tabular}

Table 4: Heat flow rate $(2 \mathcal{Q})$ in thermal creep flow of various gases between parallel plates for various $\delta$. The data in columns denoted by SB are those results from Sharipov \& Bertoldo (2009b).

for Poiseuille flows, indicate that, in the free-molecular regime, it is necessary to consider the LJ potential to get highly reliable results (Sharipov \& Strapasson 2012; Venkattraman \& Alexeenko 2012; Sharipov \& Strapasson 2013). However, the LJ model with the collision kernel (2.7) can produce mass and heat flow rates with a relative error less than $2 \%$ when $\delta>0.04$ or, equivalently, $K n<22$.

In the free molecular limit, it has previously been found that the mass flow rates in Poiseuille and thermal creep flows increase logarithmically with the Knudsen number (Cercignani \& Daneri 1963; Takata \& Funagane 2011). Our numerical results show that the heat flow rate in thermal creep flow can also be fitted to a logarithmic function of $K n$, viz, $\mathcal{Q}\left[h_{T}\right]=-0.6345 \ln (K n)-\mathcal{Q}_{0}$ in the region $10^{5}<K n<2 \times 10^{6}$, where the constant $\mathcal{Q}_{0}$ is $0.2679,0.1762,0.07371$, and -0.09903 for hard sphere, helium, argon, and the Maxwell gases when the IPL model is used, respectively.

\subsection{Poiseuille and thermal creep flow along a rectangular tube}

We now consider rarefied gases in a long straight tube that lies along the $x_{3}$ axis. The cross section is uniform and rectangular, so that $-A \ell / 2<x_{1}<A \ell / 2$ and $-\ell / 2<x_{2}<\ell / 2$, where $A$ is the aspect ratio. The linearised $\mathrm{BE}$ in dimensionless form for Poiseuille flow along this rectangular tube is:

$$
v_{1} \frac{\partial h}{\partial x_{1}}+v_{2} \frac{\partial h}{\partial x_{2}}=\mathcal{L}_{g}(h)-\nu_{e q}(v) h-v_{3} f_{e q},
$$

while $v_{3}$ should be replaced by $v_{3}\left(|\mathbf{v}|^{2}-\frac{5}{2}\right)$ for the thermal creep flow. Due to symmetry, only one quarter of the spatial domain is considered. The mass flow rate is defined as $\mathcal{M}=$ 


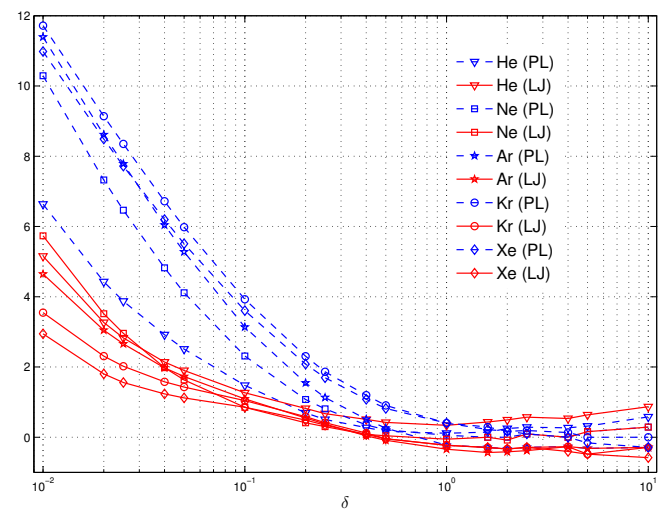

Figure 4: Relative difference in the heat flow rate between the molecular models and LJ potentials in thermal creep flow between parallel plates as a function of $\delta$.

$(4 / A) \int_{-1 / 2}^{0} \int_{-A / 2}^{0} V_{3} d x_{1} d x_{2}$ and the heat flow rate is $\mathcal{Q}=(4 / A) \int_{-1 / 2}^{0} \int_{-A / 2}^{0} q_{3} d x_{1} d x_{2}$, where $V_{3}=\int v_{3} h d \mathbf{v}$ and $q_{3}=\int\left(|\mathbf{v}|^{2}-\frac{5}{2}\right) v_{3} h d \mathbf{v}$.

This problem was first solved for a hard sphere gas by the numerical kernel method (Doi 2010) and then by the low-noise DSMC method (Radtke et al. 2011). To compare the numerical accuracy and efficiency of our new FSM, we first consider the thermal creep flow at $K n_{v h s}=0.1$ and $A=2$. We use a $50 \times 50$ nonuniform spatial grid (see equation (4.2)), $32 \times 32$ nonuniform velocity grids (equation (4.3) with $\imath=3$ ) in the $v_{1}$ and $v_{2}$ directions, and 12 uniform mesh points in the $v_{3}$ direction. The FSM with $M=6$ yields $\mathcal{M}=0.0478$, compared to 0.048 by Doi (2010) and 0.0473 by Radtke et al. (2011). We then consider Poiseuille flow in the square tube; with a $25 \times 25$ spatial cell mesh, $32 \times 32 \times 24$ frequency components, and $M=6$, we obtain $\mathcal{M}=0.3808$ and $0.3966, \mathcal{Q}=0.1365$ and 0.1874 for $K n_{v h s}=1$ and 10, respectively, compared to Doi's 0.381 and 0.396 for $\mathcal{M}$, and 0.136 and 0.187 for $\mathcal{Q}$. The computational time is 100 and 40 seconds $\dagger$, respectively, compared to the low-noise DSMC that takes 66 and 12 minutes $\ddagger$, respectively. These comparisons indicate that the FSM is an accurate and efficient new numerical method.

We observe that the difference in mass and heat flow rates between different molecular models is very small when $A=1$. Although the difference increases with $A$, at $A=10$, $\left|\left(\mathcal{M}_{A r}-\mathcal{M}_{H e}\right) / \mathcal{M}_{A r}\right|$ is only $1.6 \%$ at $\delta=0.01$, while that at $A=\infty$ is $7.6 \%$, when the LJ model is used. So, in the following numerical simulation we only use the IPL model.

We now compare our numerical results with recent experiments on the reduced mass flow rate in Poiseuille flow (Ewart et al. 2007). The tube cross section is rectangular, with an aspect ratio of $A=52.45$. The working gas is helium and we take the IPL model with $\omega=0.66$. In the spatial discretization, 100 and 50 nonuniform grid points are used in the $x_{1}$ and $x_{2}$ directions, respectively. The number of velocity grids is $32 \times 32 \times 12$, the frequency components are $32 \times 32 \times 24$, and the number of discrete angles is $M=6$. Different wall accommodation coefficients $\alpha_{a}$ are used: the wall boundary condition is given by $h\left(v_{1}, v_{2}, v_{3}, x_{1}, x_{2}=-1 / 2\right)=\left(1-\alpha_{a}\right) h\left(-v_{1}, v_{2}, v_{3}, x_{1}, x_{2}=-1 / 2\right)$ for $v_{1}>0$ and $h\left(v_{1}, v_{2}, v_{3}, x_{1}=-A / 2, x_{2}\right)=\left(1-\alpha_{a}\right) h\left(v_{1},-v_{2}, v_{3}, x_{1}=-A / 2, x_{2}\right)$ for $v_{2}>0$.

$\dagger$ Our Fortran program runs on a computer with an Intel Xeon 3.3 GHz CPU, and only one core is used.

$\ddagger$ The Fortran program runs on a single core of an Intel Q9650 (3.0 GHz Core 2 Quad processor). The time is obtained when there is better than $0.1 \%$ statistical uncertainty in the mass flow rate. To achieve the same level of uncertainty in the velocity field, the low-noise DSMC would need 240 and 120 minutes, respectively. 


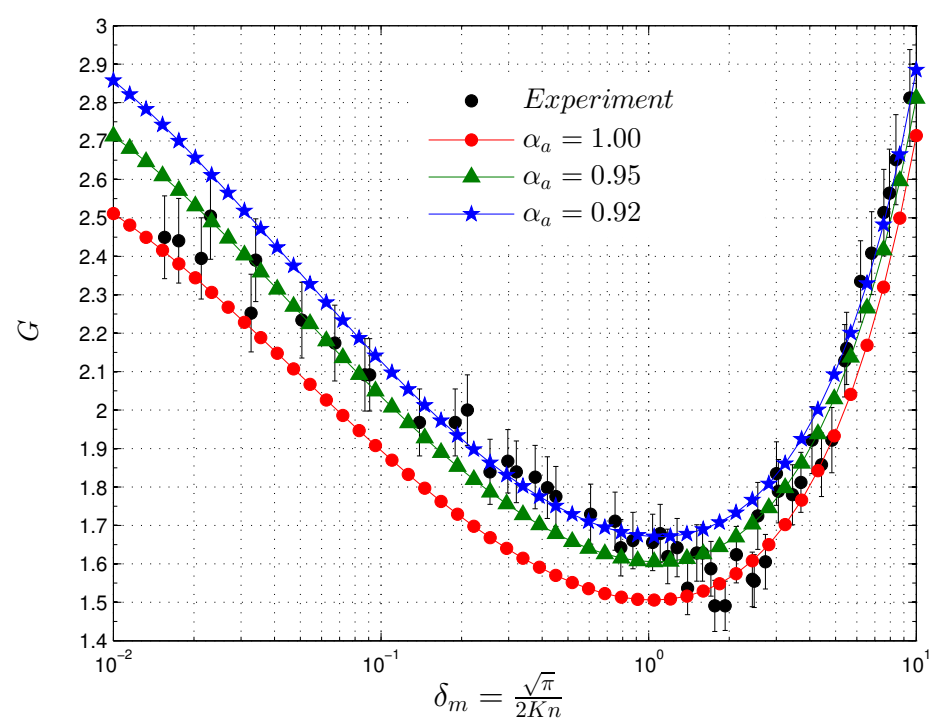

Figure 5: Comparison of the FSM-calculated reduced mass flow rate $G$ in Poiseuille flow along a rectangular tube (aspect ratio 52.45) with the experimental data of Ewart et al. (2007), for various values of the wall accommodation coefficient $\alpha_{a}$.

The obtained mass flow rate $\mathcal{M}$ is transformed to the reduced mass flow rate $G$ via the following equation (Sharipov \& Seleznev 1994):

$$
G\left(\delta_{\text {in }}, \delta_{\text {out }}\right)=\frac{2}{\delta_{\text {out }}-\delta_{\text {in }}} \int_{\delta_{\text {in }}}^{\delta_{\text {out }}} \mathcal{M}(\delta) d \delta,
$$

where the subscripts 'in' and 'out' stand for the inlet and outlet, respectively. The reduced mass flow rate $G$ no longer depends on the local pressure gradient, but only on the mean value of pressure; so the parameter $\delta_{m}$ at the mean pressure of the tube is also introduced. We consider the published experimental data where the inlet to outlet pressure ratio is five, so that $\delta_{i n}=5 \delta_{m} / 3$ and $\delta_{\text {out }}=\delta_{m} / 3$. The mass flow rate $\mathcal{M}$ is obtained at discrete values of the rarefaction parameter. The reduced mass flow rate is calculated by equation (4.8), where $\mathcal{M}$ at an unknown $\delta$ is obtained by cubic interpolation. Comparisons between the numerical and experimental data are visualized in figure 5 . When $\delta \geqslant 6$, the experimental data agrees well with the numerical results from the $\mathrm{BE}$ if $\alpha_{a}=0.92$. In the region $1 \leqslant \delta<6$, the experimental data agrees with the numerical results if $\alpha_{a}=0.92 \sim 1$. For $0.2 \leqslant \delta \leqslant 1$, the mass flow rates from the BE with $\alpha_{a}=0.92$ agree with the experimental measurements. When $\delta \leqslant 0.1$, the BE with $\alpha_{a}=0.95 \sim 1$ agrees well with the experimental results.

Note that the advantage of the FSM over the DSMC method becomes more profound for a series of simulations with the same spatial geometry but different values of $K n$. Sorting $K n$ in descending order, the converged VDF at the previous $K n$ can be used as the initial condition for the FSM for those subsequent $K n$. In this way, the computational efficiency can be improved further. For example, for this Poiseuille flow problem, it takes 4.4 hours to compute the solution for $K n=0.1$ when the initial VDF is the global equilibrium one, but only 2 hours when the initial VDF is taken as the solution at $K n=0.15$. 

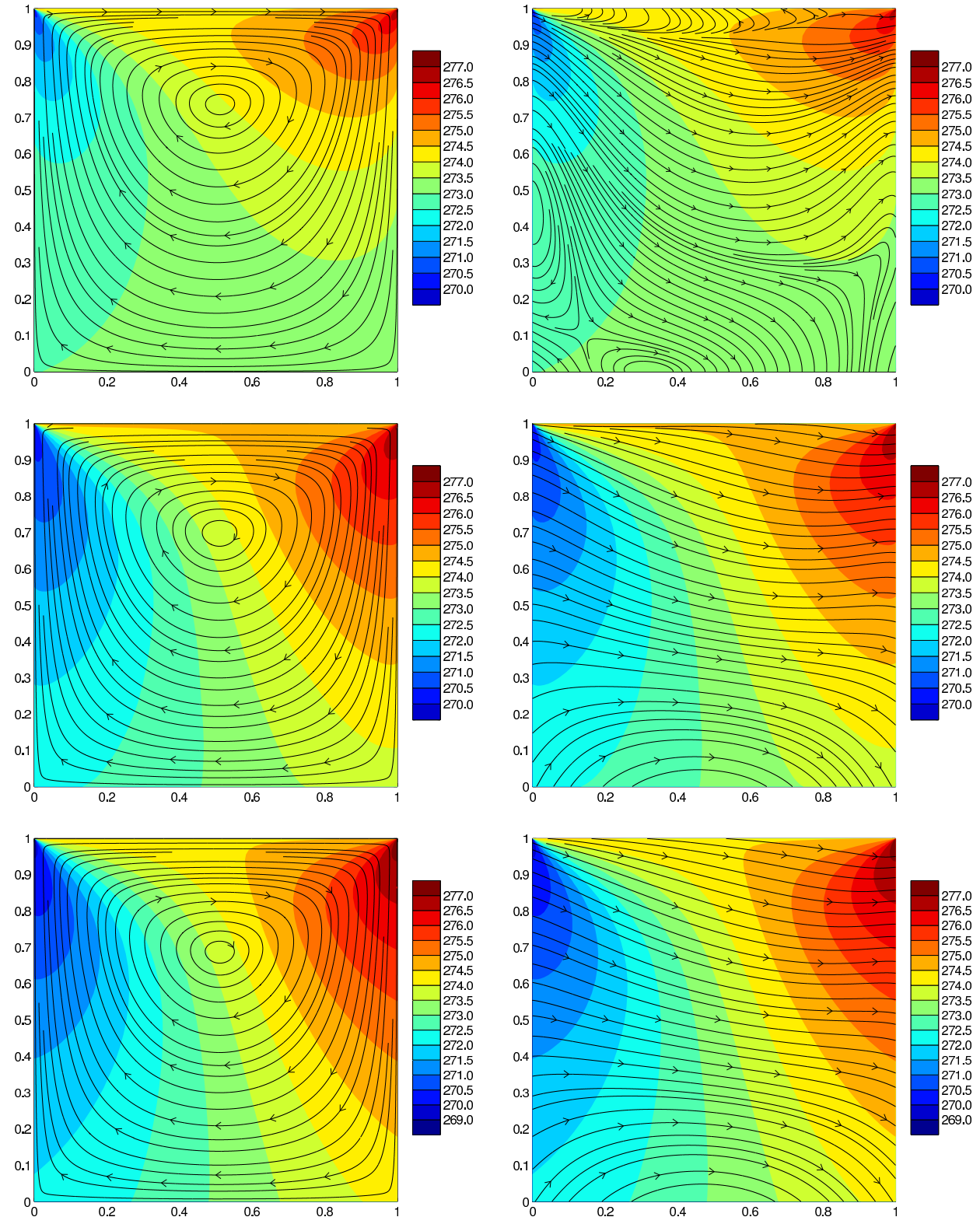

Figure 6: Temperature contours and streamlines (velocity: first column; heat flux: second column) in the lid-driven cavity flow of argon gas. From top to bottom, the Knudsen number $K n_{v h s}$ in each column is $0.1,1$, and 10, respectively. Here and after, the abscissa represents the $x_{1}$-axis, while the ordinate represents the $x_{2}$-axis.

\section{Numerical results for the nonlinear Boltzmann equation}

In this section, a number of two-dimensional simulations using the nonlinear BE are carried out in the transition flow regime $(0.1 \leqslant K n \leqslant 10)$ and the free molecular regime using the IPL models. The influence of the IPL models on the macroscopic flow properties is assessed, and the applicability of using the linear BCO in microflows with large temperature variations is evaluated. A linear scaling law is proposed for the flow veloc- 


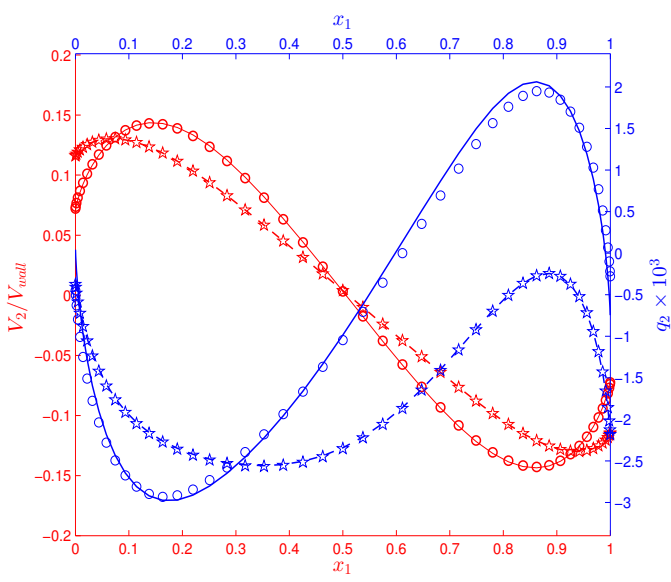

(a) At $x_{2}=0.5$

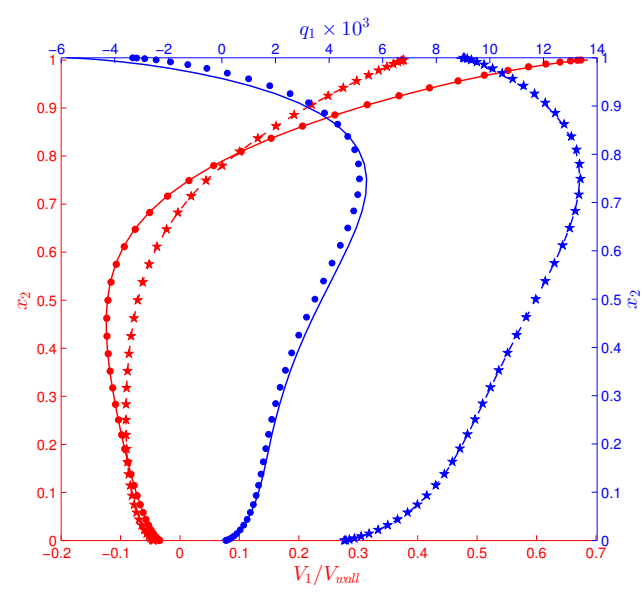

(b) At $x_{1}=0.5$

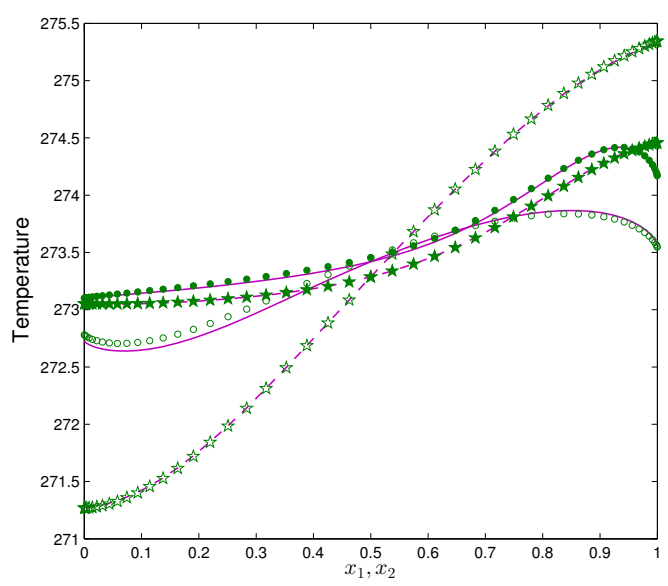

(c) Filled (open) markers: $x_{1}=0.5\left(x_{2}=0.5\right)$

Figure 7: Comparisons of the velocity, heat flux, and temperature profiles between argon and hard sphere gases in lid-driven cavity flow. The solid (or dashed) lines are the results for argon with $K n_{v h s}=0.1$ (or 10). The circles (or stars) are the results for the hard sphere gas with $K n_{v h s}=0.132$ (or 13.2).

ities in thermally driven flows in the free molecular regime. Specifically, thermal creep flow inside a closed channel is analysed, where the flow pattern can be well explained by the superposition of both the velocity profiles of linearised Poiseuille and thermal creep flow between parallel plates.

\subsection{Lid-driven cavity flow}

Consider the lid-driven flow in a square cavity, as has been previously studied using DSMC (John et al. 2010, 2011). Since the gas velocity near the lower wall is small, the DSMC solution is extremely time-consuming. Even when the velocity of the moving upper 
lid is so small that the linearised BE can be applied, the low-noise DSMC method (Radtke et al. 2011) takes more than 1 day to achieve reasonably resolved results at $K n_{v h s}=0.1$.

We solve this problem (for argon gas, the upper lid velocity of $50 \mathrm{~m} / \mathrm{s}$, and a wall temperature of $273 \mathrm{~K}$ ) in a $51 \times 51$ nonuniform spatial grid, see equation (4.2). The minimum length of the spatial cell is $7.8 \times 10^{-5}$, while the maximum is 0.0375 , with most of the grid points located near the walls. For $K n_{v h s}=0.1$ and $1,32 \times 32 \times 12$ grid points in velocity space are used, while for $K n_{v h s}=10$, there are $64 \times 64 \times 12$ velocity grid points, with most of the grid points located in $v_{1}, v_{2} \sim 0$ to capture the discontinuities in the VDF. The number of frequency components is $32 \times 32 \times 24$. The BCO is approximated by the FSM with $M=6$, and the spatial derivatives are approximated by secondorder upwind finite differences. We solve the discretized BE by the implicit scheme in an iterative manner. At the $(k+1)$-th iteration step, the VDF at the wall (entering the cavity) is determined according to the diffuse boundary condition, using the VDF at the same position (but leaving the cavity) at the previous iteration step. This numerical scheme is much faster than an explicit time-dependent technique because no CourantFriedrichs-Lewy condition is imposed (Frangi et al. 2007). However, the disadvantage to the explicit time-marching technique is that, when the mass flux entering the cavity at the $(k+1)$-th step (which is equal to that leaving the cavity at the $k$-th step) is not equal to that leaving the cavity at the $(k+1)$-th step, the total mass inside the cavity is not conserved. To overcome this, at the end of each iteration step, a simple correction of the VDF by a factor equal to the initial total mass divided by the current total mass is introduced. This is sufficient to recover the correct steady state conditions, and was introduced by Mieussens \& Struchtrup (2004).

The convergence rate of our iterative scheme is proportional to the Knudsen number. For $K n_{v h s}=0.1$ and 1, starting from the global equilibrium state, the FSM takes 110 and 14 minutes, respectively, to produce a converged solution, which is when the error between two consecutive iteration steps,

$$
\|\epsilon\|_{2}=\max \left\{\sqrt{\frac{\int\left|V_{1}^{k+1}-V_{1}^{k}\right|^{2} d x_{1} d x_{2}}{\int\left|V_{1}^{k}\right|^{2} d x_{1} d x_{2}}}, \sqrt{\frac{\int\left|V_{2}^{k+1}-V_{2}^{k}\right|^{2} d x_{1} d x_{2}}{\int\left|V_{2}^{k}\right|^{2} d x_{1} d x_{2}}}\right\},
$$

is less than $10^{-5}$.

Figure 6 shows the calculated temperature contours and streamlines in the lid-driven cavity flow of argon gas with diffuse boundary conditions. Compared to DSMC, these solutions are free of noise. We have also simulated the flow of a hard sphere gas, with the same values of the normalized wall velocity (i.e. 0.148) and shear viscosity (the Knudsen number $K n$ are the same, but $K n_{v h s}$ are different). Comparisons of the velocity, temperature, and heat flux profiles between the two IPL molecular models are shown in figure 7, and demonstrate that the molecular model has little influence on the flow pattern. This may explain why, for the same problem, the Bhatnagar-Gross-Krook and Shakhov kinetic models can both produce excellent results that are in agreement with DSMC data (Huang et al. 2012).

\subsection{Thermally driven flows}

\subsubsection{Thermal creep flow inside a closed channel}

Consider thermal creep flow in a two-dimensional closed rectangular channel with a length-to-width ratio of 5 . The temperature at the right side is set to be twice that of the left side, while the temperature of the top and bottom walls varies linearly along the channel. Using the mean density, the temperature of the left wall, and the channel width, $K n$ is set to be $0.08,0.2,0.25,0.6,2$, and 10 in the cases we investigate. Figure 8 


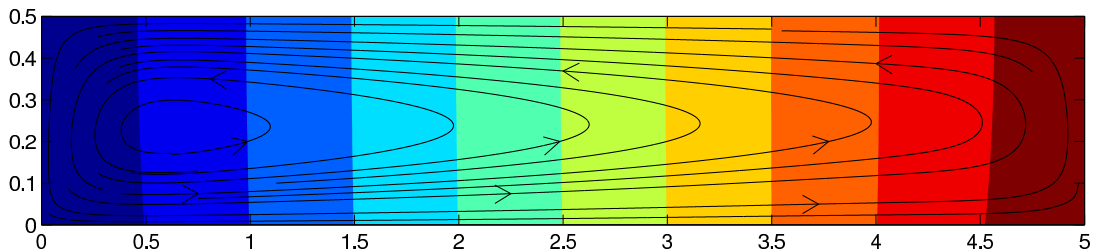

(a) $K n=0.08$

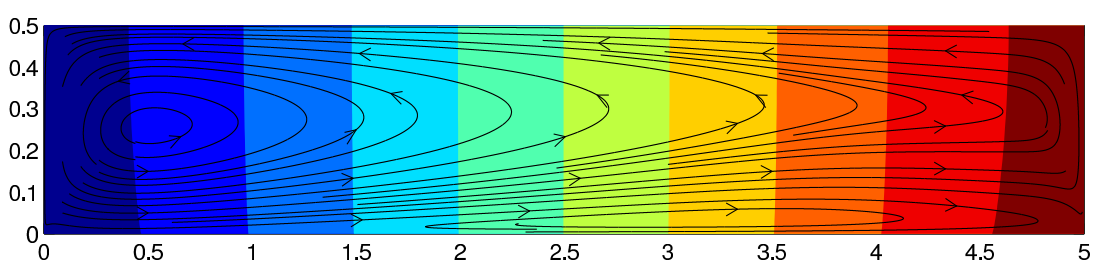

(b) $K n=0.2$

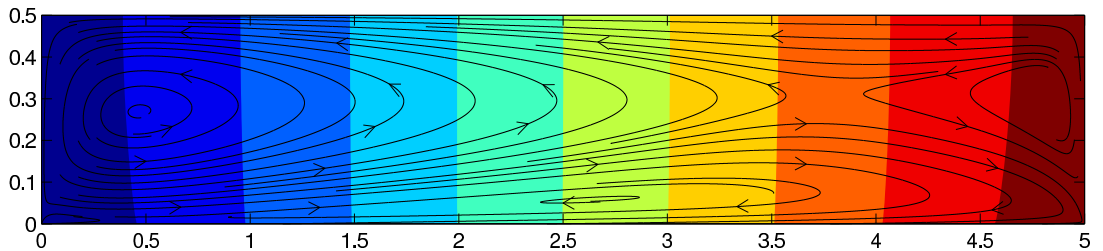

(c) $K n=0.25$

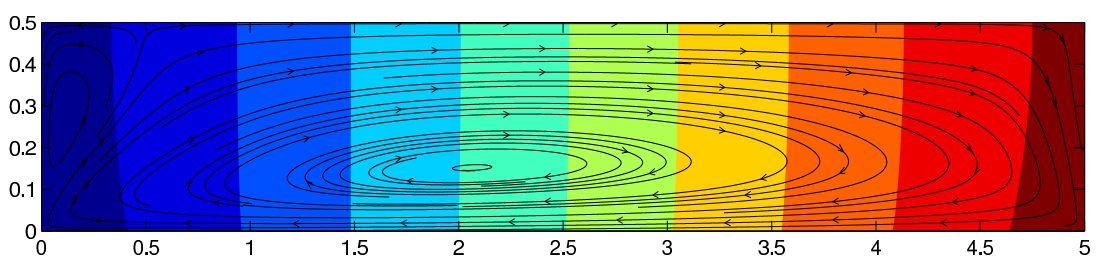

(d) $K n=0.6$

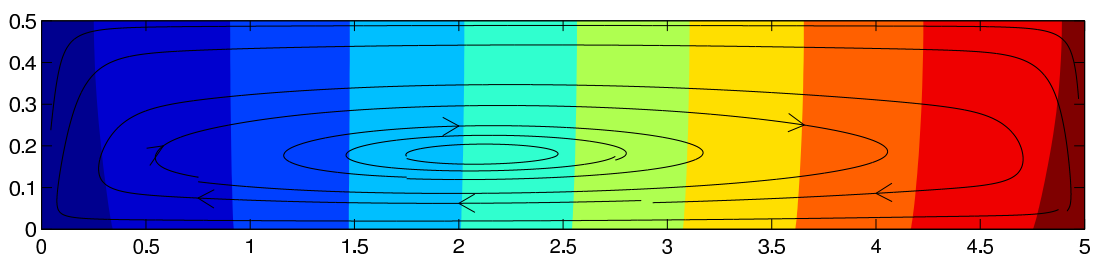

(e) $K n=2$

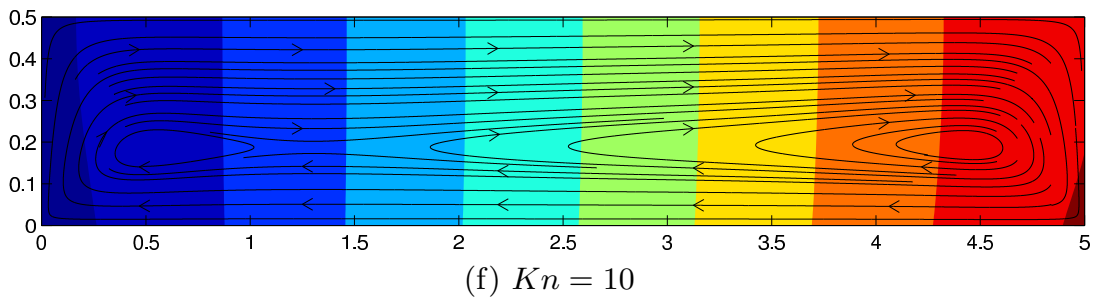

Figure 8: Temperature contours and velocity streamlines in the thermal creep flow of argon gas within a closed rectangular channel (only the down half domain is shown). In each figure, from left to right, the nondimensional temperature of each contour is $1+0.1 i$, where $i=1,2, \cdots, 9$. 


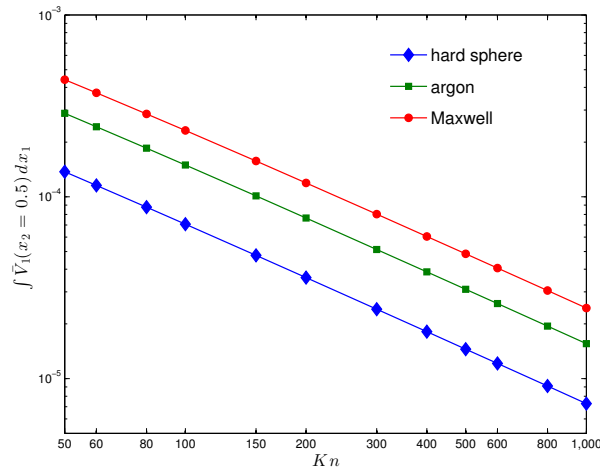

(a)

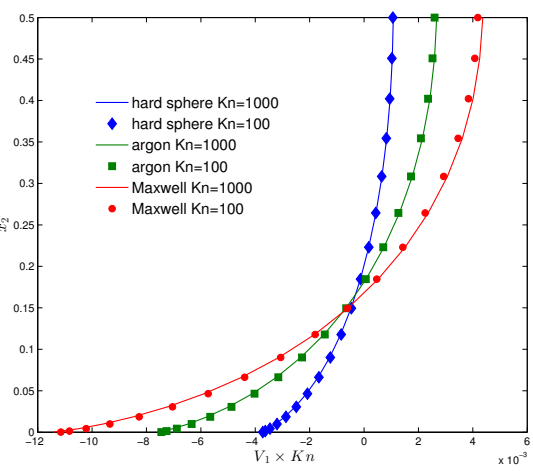

(b)

Figure 9: (a) The average horizontal velocity varying with $K n$ in thermal creep flow inside the closed rectangular channel, in the free molecular regime. In this double logarithmic diagram, the three lines have a slope of 1 , demonstrating that the velocity magnitude is proportional to $1 / K n$. (b) Examples of the linear scalability of the horizontal velocity at $x_{1}=1.4825$.

presents the resulting streamlines and the temperature distributions inside the channel for the flow of argon gas. Due to symmetry, only half of the spatial domain is shown. Unlike thermal creep in an open channel, where the flow straightforwardly moves towards the hot region, the thermal creep flow in a closed channel exhibits richer phenomena. At $K n=0.08$, the gas flows from the cold region to the hot region along the bottom wall, and returns in the central region. At $K n=0.2$, the flow still moves from hot to cold in the central region, however, near the lower wall the flow moves towards the hot region when $x_{1}<2$ and towards the cold region for at $x_{1}>2$, i.e., a circulation emerges near the lower corner of the domain. At $K n=0.25$, the circulation near the lower wall grows, which divides the flow in the central region into two circulation zones. The lower circulation zone keeps expanding, and pushes the other two circulations in the central region towards the left and right boundaries, as $K n$ increases. At $K n=0.6$, the flow direction is reversed (as compared to that when $K n=0.08$ ) and only one circulation zone remains near the left wall. The reversal of the flow direction persists but the circulations near the left wall gradually disappear as the Knudsen number increases further, for instance, to $K n=2$. By $K n=10$, the gas near the bottom wall moves from hot to cold, and two clockwise circulations emerge near the left and right sides. Finally, when the flow enters the free molecular regime, the streamline pattern does not change, but the velocity magnitudes are proportional to $1 / K n$, see figure 9 . The magnitudes of density, pressure, and temperature, however, remain unchanged irrespective of the Knudsen number.

Comparison of the velocity profiles for different molecular models at the start and the end of the transition flow regime are shown in figure 10; it can be seen that the molecular model affects the velocity magnitudes significantly.

The flow patterns shown in figure 8 can be understood qualitatively as follows. Starting from the global equilibrium state, the temperature gradient drives the gas molecules to move from cold to hot (thermal creep flow). This process increases the pressure at the right side of the channel while decreasing the pressure at the left. Then, the pressure gradient causes the gas molecules to move to the left (Poiseuille flow). The steady state is reached when the effects of Poiseuille and thermal creep flows cancel each other out, that is, when the net mass flow rate across the lines perpendicular to the top and bottom walls 


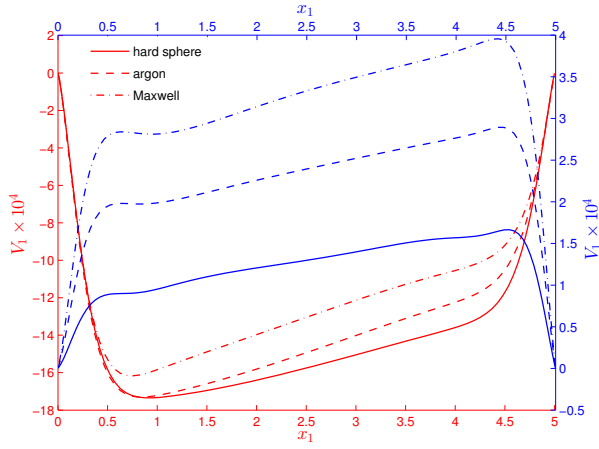

(a) Velocity along the central horizontal line

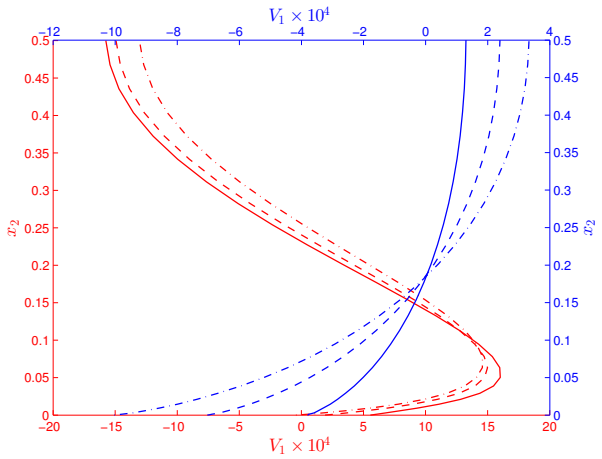

(b) Velocity along the central vertical line

Figure 10: Velocity profiles for thermal creep flow within a closed rectangular channel; $K n=0.08$ and 10 are represented by the red and blue lines, respectively.

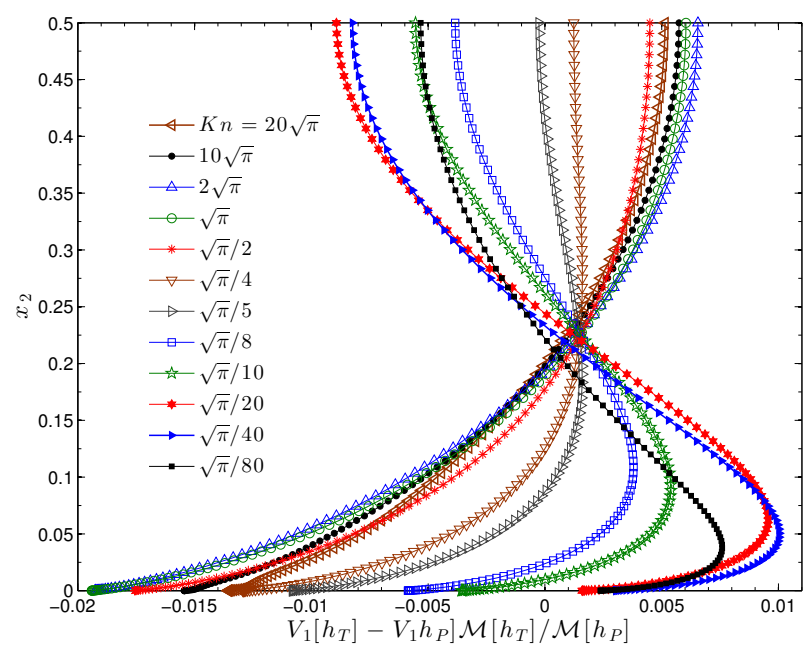

Figure 11: Net velocity profiles obtained by linear superposition of the velocity profiles of Poiseuille and thermal creep flows between parallel plates, which result in zero mass flow rate. The walls are at $x_{2}=0$ and $x_{2}=1$ and the working gas is argon, where the IPL model with $\omega=0.81$ is used.

is zero. The horizontal velocity profile can be analysed by assuming the wall temperature gradient to be small (i.e. the channel is long enough) so that the BE can be linearised. In this case, we can directly use the velocity profiles obtained in Sec. 4. Figure 11 plots the net velocity profiles in the linear superposition of Poiseuille and thermal creep flows between parallel plates where the net mass flow rate is zero. The flow velocities are normalised; in real problems, the horizontal velocity is given by (see the first paragraph in Sec. 4.2):

$$
V_{1}=\beta_{T}\left\{V_{1}\left[h_{T}\right]-\frac{\mathcal{M}\left[h_{T}\right]}{\mathcal{M}\left[h_{P}\right]} V_{1}\left[h_{P}\right]\right\},
$$

where $\beta_{T}$ is the temperature gradient; in this case, it is about $1 / 5$.

Now the horizontal velocity profiles in figure 11 can be used to explain the flow patterns in figure 8. From figure 11 we find that, when $K n=\sqrt{\pi} / 20$, the net horizontal velocity is 

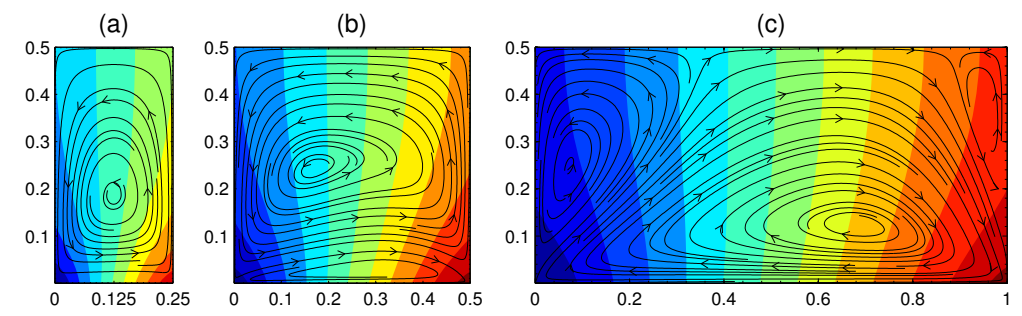

(d)

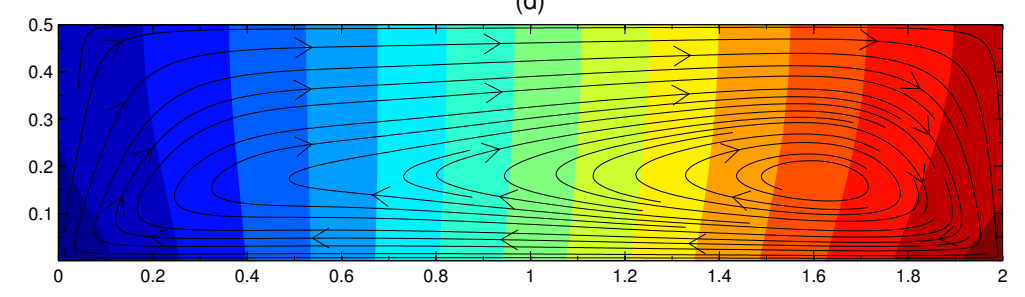

Figure 12: Thermal creep flow patterns of the argon gas (IPL model with $\omega=0.81$ ) in the free molecular regime at different values of length-to-height ratio $A$. (a) $A=0.25$, (b) $A=0.5$, (c) $A=1$, and (d) $A=2$.

positive when $x_{2}<0.25$ and negative otherwise, which agrees well with the flow pattern in figure $8(\mathrm{a})$. Also, from figure 10 (a) we find that, at $K n=0.08, V_{1}\left(x_{2}=0.5\right) \simeq-0.0018$ near the left side of the channel, which is well predicted by equation (5.2) when we choose $K n=\sqrt{\pi} / 20$ and $x_{2}=0.5: V_{1}\left(x_{2}=0.5\right) \simeq(-0.009) / 5=-0.0018$. Furthermore, as $K n$ increases, the magnitude of the horizontal velocity at $x_{2}=0.5$ decreases (figure 11). This is in accordance with the horizontal velocity profiles shown in figure 10(a), where the velocity magnitude decreases as $x_{1}$ increases from 1 to 4 , because the local Knudsen number increases along the channel as a result of increasing temperature and decreasing particle number density. When $K n=\sqrt{\pi} / 10$ (and $\sqrt{\pi} / 8$ ), the net horizontal velocity in figure 11 is positive when $0.26>x_{2}>0.009$ (and $0.28>x_{2}>0.023$ ) and negative otherwise. This explains the flow patterns at the right side of the channel, as shown in figure 8 (b). As $K n$ increases, the extent of the region near the bottom wall where the velocity is negative increases (figure 11), so that the circulation near the bottom wall in figure $8(\mathrm{c})$ is larger than that in figure $8(\mathrm{~b})$. When $K n$ increases above a critical value of around $\sqrt{\pi} / 5$, the horizontal velocity in figure 11 is negative when $x_{2}$ is smaller than some fixed value $x_{2 c}$ and positive otherwise. In this case, the flow direction is completely reversed in comparison with that at small Knudsen numbers. When $K n>\sqrt{\pi} / 2$, the fixed value is $x_{2 c}=0.2$. In figure $8(\mathrm{~d}-\mathrm{f})$, we see that the gas moves from left to right if $x_{2}<0.2$, and moves right to left if $x_{2}>0.2$.

Note that the above analysis is valid at positions far from the left and right walls; near the ends of the channel, the horizontal velocity is nearly zero and the above analysis fails. This end effect becomes dominant in the whole channel when the molecular mean free path is of the order of half the distance between the left and right walls. When the mean free path is much larger than the wall distance, from figures 8 and 9 we find that the streamline pattern does not change very much, but the velocity magnitude is inversely proportional to the Knudsen number. In other words, at large Knudsen numbers, the flow pattern is determined by the velocity profiles in figure 11 at a critical Knudsen number. In our numerical simulations, we find that the critical Knudsen number varies linearly 
with the length-to-height ratio $A$ of the channel:

$$
K n_{c} \simeq 0.35 \mathrm{~A} .
$$

For instance, when $A=0.25$, the end effect becomes dominant when $K n_{c} \geqslant 0.09$, and the flow pattern at $K n \gg K n_{c}$ is similar to the flow pattern at $K n_{c}$. Figure 11 shows that at $K n=0.09$ the molecules move from left to right near the bottom wall, and return to the left at $x_{2}=0.5$, which is exactly the case shown in figure 12 (a). For $A=0.5$, $K n_{c}=0.18 \simeq \sqrt{\pi} / 10$, and from figure 11 we see the horizontal flow velocity turns from negative to positive and then back to negative as we move from the bottom wall to the central region, which is the same as in figure $12(\mathrm{~b})$. The aspect ratio $A=1$ is a critical case, since near $K n_{c}=0.35 \simeq \sqrt{\pi} / 5$, the horizontal velocity at $x_{2}=0.5$ could be negative or positive, depending on whether the local Knudsen number is smaller or larger than $\sqrt{\pi} / 5$. That is why the flow pattern shown in figure 12(c) is more complicated. For $A=2$, where $K n_{c}=0.7$, the flow pattern is simpler, and the molecules move from the hot to the cold region near the bottom and return from the cold to the hot region near $x_{2}=0.5$, see figure $12(\mathrm{~d})$. For $K n<K n_{c}$, the horizontal velocity profiles can be analysed using the data in figure 11.

\subsubsection{Flow induced by a spatially-periodic wall temperature}

Consider the gas flow between two parallel plates that have spatially-periodic temperature: the upper $\left(x_{2}=\ell\right)$ and lower plates $\left(x_{2}=0\right)$ have the temperature $T_{0}(1-$ $\left.0.5 \cos 2 \pi x_{1}\right)$. Due to symmetry, the spatial domain is chosen as $0 \leqslant x_{1}, x_{2} \leqslant 1 / 2$. The specular reflection boundary condition is chosen for the left, upper, and right boundaries, while the diffuse boundary condition is employed at the lower wall. Using the mean density, mean temperature $T_{0}$, and the wall distance $\ell$, the unconfined Knudsen number $K n$ is chosen to be $0.1,1$, and 10 . In the spatial discretization, 51 equispaced points are used in the $x_{1}$ direction and 51 non-uniform points (see equation (4.2)) are placed in the $x_{2}$ direction, with most of the points close to the lower wall. In the discretization of the velocity space, $64 \times 64 \times 16$ (maximum velocity $L=7.5, L_{2}=5$ in equation (4.3) with $\imath=3$ ) grid points are used when $K n=0.1$ and 1 , while $128 \times 128 \times 16$ grid points are used when $K n=10$. The number of frequency components is $32 \times 32 \times 32$. Even with such a large number of velocity grid points, the FSM with $M=6$ takes only about 150 minutes to converge to $\|\epsilon\|_{2}$ less than $10^{-5}$ at $K n=0.1$.

The temperature contours and velocity streamlines are shown in figure 13. The gas moves from the cold to the hot region near the lower wall, while it returns from hot to cold around the central horizontal region. The circulation centre approaches the lower wall as $K n$ increases. The marginal VDFs, which become more complicated as $K n$ increases, are shown in figure 14, and large discontinuities at the lower wall and fine structures are clearly seen. This demonstrates the necessity of using a large number of velocity grid points in the $v_{1}$ and $v_{2}$ directions at large $K n$, in order to get a high resolution. The flow velocities for different molecular models are compared in figure 15: the maximum velocity increases with the viscosity index. We have also investigated the flow in the free molecular regime and find that the velocity magnitudes are inversely proportional to the Knudsen number, as in the case of thermal creep flow inside closed channels.

Note that this problem has also been solved by the low-noise DSMC method, based on the linearised BE (Radtke et al. 2011): from figure 14 we see that the VDFs deviate significantly from the equilibrium state, so the required linearisation around the global equilibrium state may not be appropriate. We test this by solving the linearised BE using the same spatial and velocity grids. Although the velocity is very small, the numerical comparisons shown in figure 15 indicate that linearisation around the global state does 


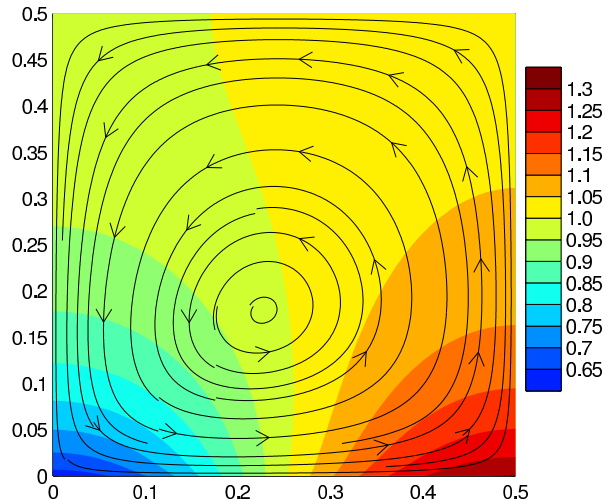

(a) $K n=0.1$

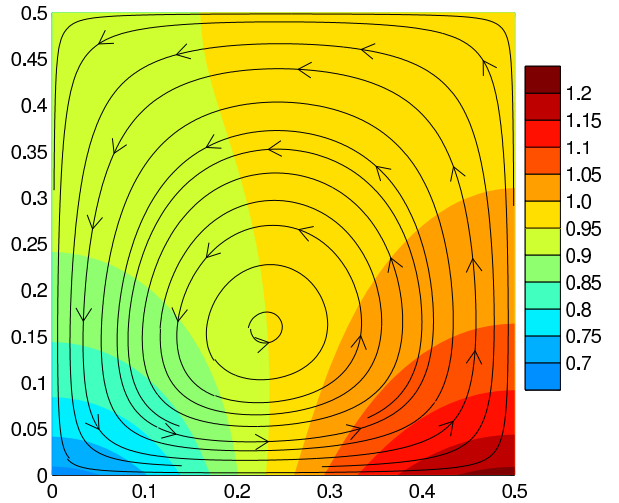

(b) $K n=1$

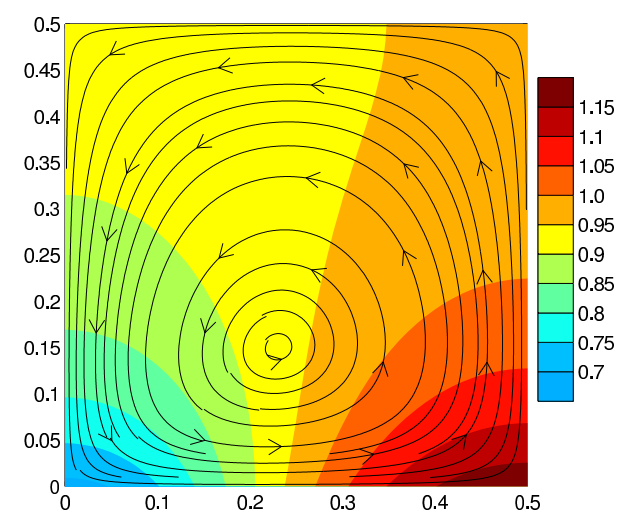

(c) $K n=10$

Figure 13: Contour plots of the temperature, and the velocity streamlines in argon gas subjected to a spatially-periodic wall temperature.
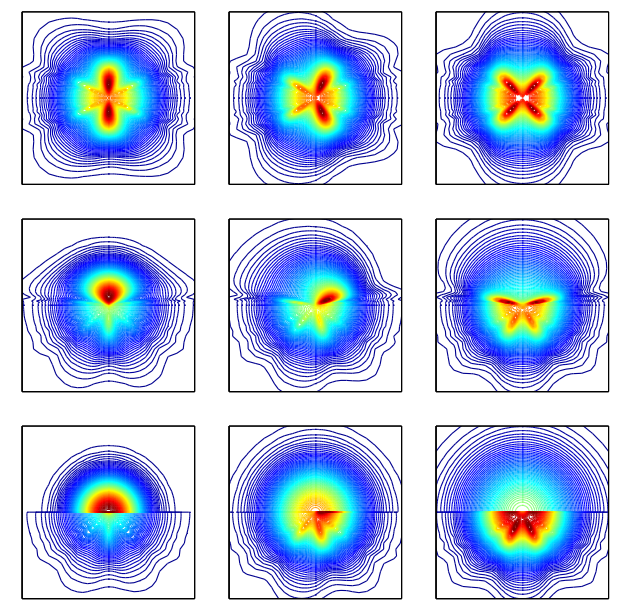

(a) $K n=1$

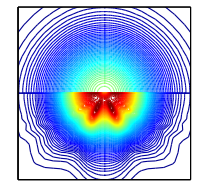

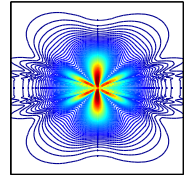
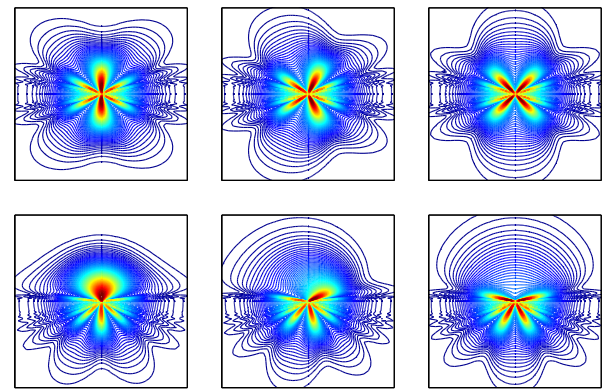
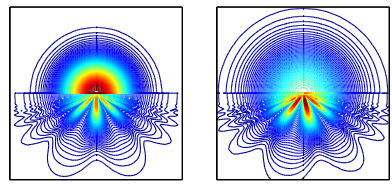

(b) $K n=10$

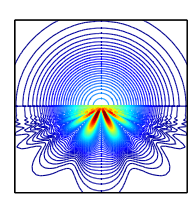

Figure 14: Contour plots of the marginal VDF, $\int f d v_{3}$, for $K n=1$ and $K n=10$. In each figure, from bottom to top, $x_{2}=0.5,0.25$, and 0.5 , respectively. From left to right, $x_{1}=0,0.25$, and 0.5 , respectively. The velocity region shown is $[-2,2] \times[-2,2]$. 


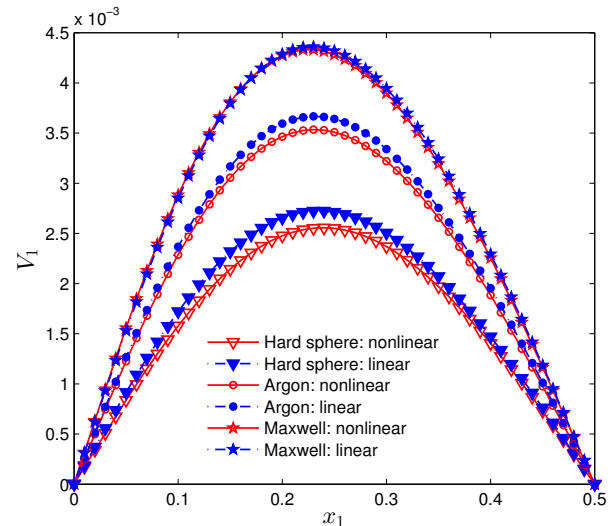

(a) At $x_{2}=0$

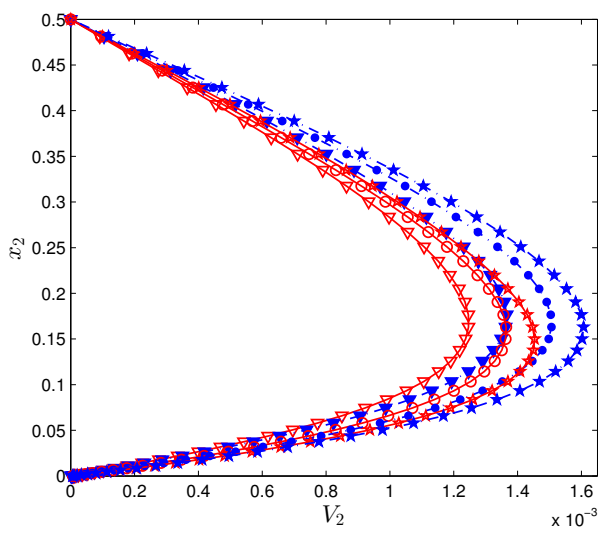

(b) At $x_{1}=0.5$

Figure 15: Comparisons of the velocity profiles predicted by the full nonlinear and the linearised BEs, and for different molecular models. The unconfined Knudsen number is $K n=1$ and the temperature of the top and bottom walls vary as $T_{0}\left(1-0.5 \cos 2 \pi x_{1}\right)$.

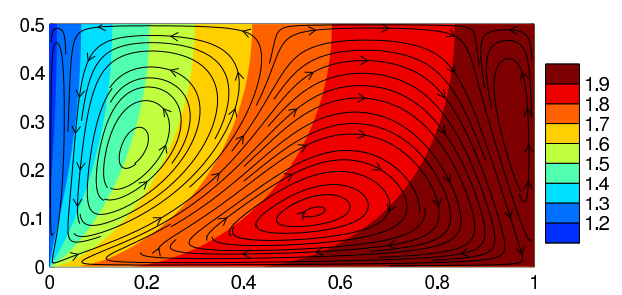

(a) $K n=0.1$

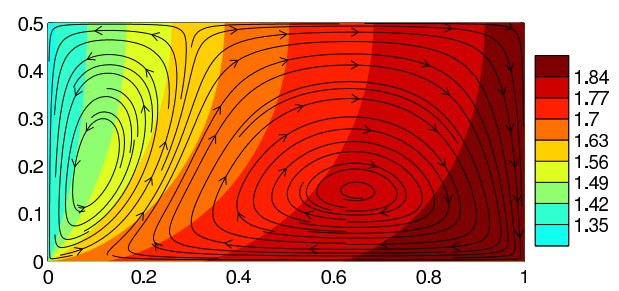

(b) $K n=1$

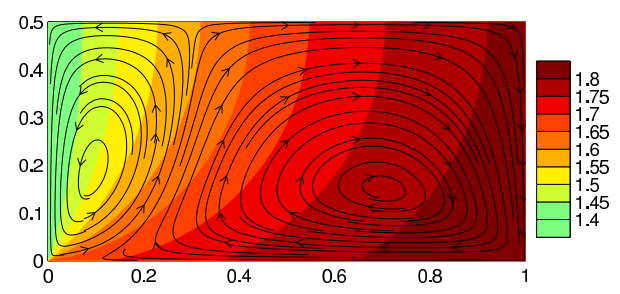

(c) $K n=10$

Figure 16: Temperature contours and velocity streamlines in the flow in a square box of a Maxwell gas driven by a temperature discontinuity at the bottom left corner.

not give accurate results in this problem if the variation of the wall temperature is strong. If the temperature variation is weak, e.g. the wall temperature is $T_{0}\left(1-0.05 \cos 2 \pi x_{1}\right)$, however, the linearised BE can be used effectively.

\subsubsection{Flow induced by a temperature discontinuity}

Finally, we consider the gas flow inside a square box that is driven by a temperature discontinuity: the temperature of the left wall is one half that of the other three walls. In terms of the mean density, temperature of the left wall, and the wall distance, $K n$ is 0.1 , 1 , and 10 in the cases we investigate. The half spatial region $\left(0 \leqslant x_{1} \leqslant 1,0 \leqslant x_{2} \leqslant 0.5\right)$ is simulated, due to symmetry. The nonuniform spatial grids are $101 \times 100$ for $K n=0.1$, and $51 \times 30$ for $K n=1$ and 10 . The velocity grids are $48 \times 48 \times 12$ for $K n=0.1$ 


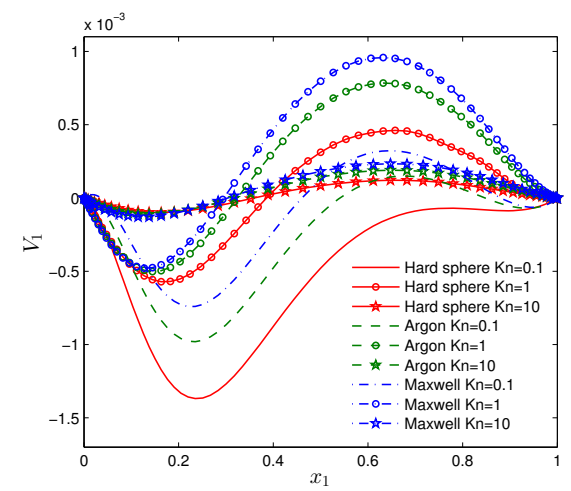

(a) velocity along the central horizontal line

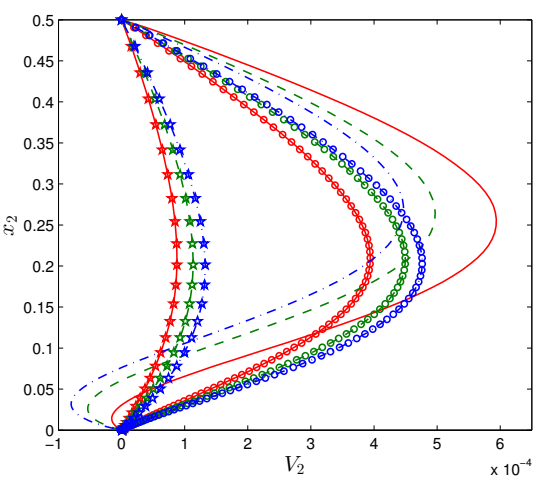

(b) velocity along the central vertical line

Figure 17: Comparison of the velocity profiles for different molecular models for the flow in a square box driven by a temperature discontinuity at the corner.

and 1 , and $64 \times 64 \times 12$ for $K n=10$. The resulting temperature contours and velocity streamlines for a Maxwell gas in this configuration are shown in figure 16 . At $K n=0.1$, four circulation zones arise. As $K n$ increases, the two circulations near the left and the right walls gradually disappear, while the centre of the largest circulation moves towards the right wall. Note that this problem was previously studied using the original DSMC method (Huang et al. 2013), and comparison shows that our FSM yields much smoother streamlines than the DSMC method at $K n=0.1$.

The velocity profiles along the central horizontal and vertical lines are depicted in figure 17, and clearly show the influence of different molecular models. However, interestingly, the molecular model has little effect on the temperature and heat flux profiles (not shown). We have also solved this problem using the BGK model, and found that while the BGK model gives almost identical temperature and heat flux profiles, it cannot recover the velocity profiles.

In the free molecular limit, the density, pressure, and temperature profiles reach fixed values independent of the Knudsen number. The streamline pattern also remains unchanged, except that the velocity magnitudes decrease as $1 / K n$.

\section{Conclusions}

We have studied the application and usefulness of the Fast Spectral Method (FSM) for gas microflow simulations. A numerical approximation to both the linearised and nonlinear Boltzmann collision operator was presented. Numerical accuracy has been evaluated by comparing our FSM results with the numerical kernel method for Poiseuille flow, and excellent agreements in the mass and heat flow rates were seen up to $K n \sim 10^{6}$. Computational efficiency has been demonstrated on two-dimensional Poiseuille and liddriven cavity flows, and we found that the FSM can be 10 to 50 times faster than the low-noise DSMC method (which itself is much faster than the original DSMC technique for microflow simulations).

We have investigated the influence of different molecular models on the mass and heat flow rates in Poiseuille and thermal creep flows between parallel plates. The mechanism of the variability in flow rates for different molecular models with the same value of shear viscosity is explained in terms of the effective collision frequency. We also assessed the accuracy of special collision kernels for the LJ potential, and found that these can 
produce mass and heat flow rates with a relative error of less than $2 \%$ for $K n<22$ and about $5 \%$ at $K n \simeq 88$, when compared to results obtained using the realistic LJ potential; for the flow through a rectangular channel, this relative error decreases as the aspect ratio decreases from infinity to 1 .

We have simulated a number of two-dimensional microflows. New insight into experimental mass flow rates along a rectangular tube with a large aspect ratio (Ewart et al. 2007) has been provided. We have also analysed quantitatively the influence of different molecular models on the flow properties. In lid-driven cavity flow, we found that the solution is only determined by the value of the shear viscosity, irrespective of the molecular model. For thermally driven flows, however, the molecular model affects the flow velocity significantly, but the temperature field is not very sensitive to the molecular model. In the free molecular regime, our numerical results showed that the streamline pattern does not change but the velocity magnitudes are inversely proportional to the Knudsen number. We have also analysed in detail the thermal creep flow inside a closed rectangular channel, and found that the streamline pattern can be predicted based on the velocity profiles of superposed Poiseuille and thermal creep flows between parallel plates. These thermally driven flows can serve as benchmark cases for future investigations.

Finally, we brief analyse why the FSM is suitable for the simulation of moderately and/or highly rarefied microflows. The molecular velocity distribution functions have large discontinuities at large $K n$, hence a significant number of velocity grids are needed. This poses an extremely difficult problem for other deterministic methods that handle binary collisions in velocity space. However, this problem becomes amenable for the FSM because the collisions are treated in frequency space. The FSM approximates the collision operator with spectral accuracy (Mouhot \& Pareschi 2006); the number of frequency components does not need to be as large as the velocity grids. One reason for this is that discontinuities in the distribution function produce high frequency components in its spectrum (and this is usually smooth, or at least smoother than the distribution function); in the calculation of the spectrum of the collision operator, the spectrum of the distribution function is multiplied by a weight function which is very small for high frequency components (see figure 4 in Wu et al. (2013)). Therefore, very high frequencies can be safely ignored: in the transition flow regime, we have shown that 32 frequency components in each direction is enough.

The only drawback of the FSM, like all other deterministic numerical methods, is that a large amount of compute memory is required (relative to that required for the DSMC method).

\section{Acknowledgements}

YHZ thanks the UK's Royal Academy of Engineering (RAE) and the Leverhulme Trust for the award of a RAE/Leverhulme Senior Research Fellowship. This work is financially supported by the UK's Engineering and Physical Sciences Research Council (EPSRC) under grants EP/I036117/1 and EP/I011927/1. The authors also thank the reviewers of this paper for their helpful comments.

\section{REFERENCES}

Baker, L. L. \& Hadjiconstantinou, N. G. 2005 Variance reduction for Monte Carlo solutions of the Boltzmann equation. Phys. Fluids 17 (5), 051703.

BIRD, G. A. 1994 Molecular Gas Dynamics and the Direct Simulation of Gas Flows. Oxford University Press Inc, New York: Oxford Science Publications. 
Cercignani, C. 1990 Mathematical Methods in Kinetic Theory. 223 Spring Street, New York, N.Y. 10013: Plenum Publishing Inc.

Cercignani, C. \& Daneri, A. 1963 Flow of a rarefied gas between two parallel plates. J. Appl. Phys. 34, 3509 .

Chapman, S. \& Cowling, T.G. 1970 The Mathematical Theory of Non-uniform Gases. Cambridge University Press.

DoI, T. 2010 Numerical analysis of the Poiseuille flow and thermal transpiration of a rarefied gas through a pipe with a rectangular cross section based on the linearized Boltzmann equation for a hard sphere molecular gas. J. Vac. Sci. Technol. A 28, 603-612.

DoI, T. $2012 a$ Effect of weak gravitation on the plane Poiseuille flow of a highly rarefied gas. Zeitschrift für angewandte Mathematik und Physik 63, 1091-1102.

DoI, T. $2012 b$ Plane thermal transpiration of a rarefied gas in the presence of gravitation. Vacuum 86, 1541-1546.

Ewart, T., Perrier, P., Graur, I. A. \& Méolans, J. G. 2007 Mass flow rate measurements in a microchannel, from hydrodynamic to near free molecular regimes. J. Fluid Mech. 584, $337-356$.

FAn, J. \& Shen, C. 2001 Statistical simulation of low-speed rarefied gas flows. J. Comput. Phys. 167, 393-412.

Frangi, A., Ghisi, A. \& Frezzotti, A. 2007 Analysis of gas flow in MEMS by a deterministic 3D BGK kinetic model. Sens. Lett. 6 (1), 1-7.

Funagane, H. \& TAKata, S. 2012 Hagen-Poiseuille and thermal transpiration flows of a highly rarefied gas through a circular pipe. Fluid Dyn. Res. 44, 055506.

Gamba, I. M. \& Tharkabhushanam, S. H. 2009 Spectral-Lagrangian methods for collisional models of non-equilibrium statistical states. J. Comput. Phys. 228 (6), 2012-2036.

Garcia-Colin, L. S., Velasco, R. M. \& Uribe, F. J. 2008 Beyond the Navier-Stokes equations: Burnett hydrodynamics. Phys. Rep. 465, 149-189.

Gu, X. J. \& Emerson, D. R. 2009 A high-order moment approach for capturing nonequilibrium phenomena in the transition regime. J. Fluid Mech. 636, 177-216.

Homolle, T. M. M. \& Hadjiconstantinou, N. G. 2007 A low-variance deviational simulation Monte Carlo for the Boltzmann equation. J. Comput. Phys. 226 (2), 2341-2358.

HuAng, J. C., Xu, K. \& Yu, P. B. 2012 A unified gas-kinetic scheme for continuum and rarefied flows II: Multi-dimensional cases. Commun. Comput. Phys. 12, 662-690.

Huang, J. C., Xu, K. \& Yu, P. B. 2013 A unified gas-kinetic scheme for continuum and rarefied flows III: Microflow simulations. Commun. Comput. Phys. 14, 1147-1173.

John, B., Gu, X. J. \& Emerson, D. R. 2010 Investigation of heat and mass transfer in a liddriven cavity under nonequilibrium flow conditions. Num. Heat Transfer B 52, 287-303.

John, B., Gu, X. J. \& Emerson, D. R. 2011 Effects of incomplete surface accommodation on non-equilibrium heat transfer in cavity flow: A parallel DSMC study. Computers $\&$ Fluids 45, 197-201.

Loyalka, S. K. 1971 Kinetic Theory of Thermal Transpiration and Mechanocaloric Effect. I. J. Chem. Phys. 55, 4497.

Masters, N. D. \& Ye, W. J. 2007 Octant flux splitting information preservation DSMC method for thermally driven flows. J. Comput. Phys. 226 (2), 2044-2062.

Mieussens, L. \& Struchtrup, H. 2004 Numerical comparison of Bhatnagar-Gross-Krook models with proper Prandtl number. Phys. Fluids 16 (8), 2297-2813.

Mounot, C. \& PAReschi, L. 2006 Fast algorithms for computing the Boltzmann collision operator. Math. Comput. 75 (256), 1833-1852.

Ohwada, T., Sone, Y. \& Aoki, K 1989 Numerical analysis of the Poiseuille and thermal transpiration flows between two parallel plates on the basis of the Boltzmann equation for hard sphere molecules. Phys. Fluids A 1, 2042.

Pareschi, L. \& Russo, G. 2000 Numerical solution of the Boltzmann equation I: Spectrally accurate approximation of the collision operator. SIAM J. Numerical Analysis 37 (4), $1217-1245$.

Radtke, G. A., Hadjiconstantinou, N. G. \& Wagner, W. 2011 Low-noise Monte Carlo simulation of the variable hard sphere gas. Phys. Fluids 23 (3), 030606.

Rana, A., Torrilhon, M. \& Struchtrup, H. 2013 A robust numerical method for the R13 
equations of rarefied gas dynamics: Application to lid driven cavity. J. Comput. Phys. 236, $169-186$.

Sharipov, F. 1994a Onsager-Casimir reciprocity relations for open gaseous systems at arbitrary rarefaction. I. General theory for single gas. Physica A 203, 437-456.

Sharipov, F. $1994 b$ Onsager-Casimir reciprocity relations for open gaseous systems at arbitrary rarefaction. II. Application of the theory for single gas. Physica A 203, 457-485.

Sharipov, F. \& Bertoldo, G. $2009 a$ Numerical solution of the linearized Boltzmann equation for an arbitrary intermolecular potential. J. Comput. Phys. 228, 3345-3357.

Sharipov, F. \& Bertoldo, G. $2009 b$ Poiseuille flow and thermal creep based on the Boltzmann equation with the Lennard-Jones potential over a wide range of the Knudsen number. Phys. Fluids 21, 067101.

Sharipov, F. \& Seleznev, V. 1994 Rarefied flow through a long tube at any pressure ratio. J. Vac. Sci. Technol. A 12 (5), 2933-2935.

Sharipov, F. \& Seleznev, V. 1998 Data on internal rarefied gas flows. J. Phys. Chem. Ref. Data 27 (3), 657-706.

Sharipov, F. \& Strapasson, J. L. 2012 Ab initio simulation of transport phenomena in rarefied gases. Phys. Rev. E 86, 031130.

Sharipov, F. \& Strapasson, J. L. 2013 Benchmark problems for mixtures of rarefied gases. I. Couette flow. Phys. Fluids 25, 027101.

TAKATA, S. $2009 a$ Note on the relation between thermophoresis and slow uniform flow problems for a rarefied gas. Phys. Fluids 21, 112001.

TAKatA, S. $2009 b$ Symmetry of the linearized Boltzmann equation and its application. J. Stat. Phys. 136, 751

TAKATA, S. \& FunAgane, H. 2011 Poiseuille and thermal transpiration flows of a highly rarefied gas: over-concentration in the velocity distribution function. J. Fluid Mech. 669, 242-259.

Venkattraman, A. \& Alexeenko, A. A. 2012 Binary scattering model for Lennard-Jones potential: Transport coefficients and collision integrals for non-equilibrium gas flow simulations. Phys. Fluids 24, 027101.

Wu, L., White, C., Scanlon, T. J., Reese, J. M. \& Zhang, Y. H. 2013 Deterministic numerical solutions of the Boltzmann equation using the fast spectral method. J. Comput. Phys. 250, 27-52. 